ESAIM: M2AN

Vol. 41, No 6, 2007, pp. 1061-1087

DOI: $10.1051 / \mathrm{m} 2 \mathrm{an}: 2007050$
ESAIM: Mathematical Modelling and Numerical Analysis

www.esaim-m2an.org

\title{
SMALL AMPLITUDE HOMOGENIZATION APPLIED TO MODELS OF NON-PERIODIC FIBROUS MATERIALS
}

\author{
DaVid MANCEAU ${ }^{1}$
}

\begin{abstract}
In this paper, we compare a biomechanics empirical model of the heart fibrous structure to two models obtained by a non-periodic homogenization process. To this end, the two homogenized models are simplified using the small amplitude homogenization procedure of Tartar, both in conduction and in elasticity. A new small amplitude homogenization expansion formula for a mixture of anisotropic elastic materials is also derived and allows us to obtain a third simplified model.
\end{abstract}

Mathematics Subject Classification. 35J25, 74Q15, 74B05.

Received November 10, 2006. Revised June 29, 2007.

\section{INTRODUCTION}

The left ventricle of the heart is composed of oriented fibers. Anatomic studies show that the cardiac fibers have an orientation that varies continuously from an angle $\gamma_{0}$ at the endocardium to $-\gamma_{0}$ at the epicardium. Several biomechanics empirical models were derived (see e.g. Arts [3], Chadwick [10], Feit [11], Peskin [17] and Streeter [20]) considering the fibers as an oriented elastic material embedded in a homogeneous medium. In particular, Peskin [17] deduced this fiber architecture of the heart from the starting assumption that the stress matrix reads as

$$
\sigma:=\sigma_{m}+\sigma_{f}, \quad \text { with } \quad \sigma_{f}:=T\left(e_{f}\right)(\tau \otimes \tau),
$$

where $\sigma_{m}$ is the isotropic medium stress matrix, $\sigma_{f}$ is the stress matrix in the fiber direction, $\tau$ is the fiber direction, $T\left(e_{f}\right)$ the fiber tension and $e_{f}$ the strain coefficients in the fiber direction. Then, in linear elasticity this general biomechanics model leads us to a stress matrix of the type

$$
\sigma:=\lambda \operatorname{tr}(e) I_{3}+2 \mu e+T\left(e_{f}\right)(\tau \otimes \tau)
$$

where $\lambda, \mu$ are the Lamé coefficients of the medium and $e$ is the strain matrix.

In conduction, the strain matrix $e$ of $(0.2)$ is replaced by the electric field $\nabla u$, and the stress matrix $\sigma$ by the electrical current $A \nabla u$, where $A$ is the conductivity matrix of the composite material. Then, the analogue

\footnotetext{
Keywords and phrases. Non-periodic homogenization, fibrous material, small amplitude, low contrast, conduction, linear elasticity, $H$-measures.

1 Centre de Mathématiques, I.N.S.A. de Rennes \& I.R.M.A.R., 20 avenue des Buttes de Coëmes, CS 14315 - 35043 Rennes Cedex, France. dmanceau@insa-rennes.fr 
of (0.2) in conduction leads us to the conductivity matrix

$$
A:=\alpha I_{3}+\beta(\tau \otimes \tau) .
$$

This model is based on the two following assumptions: the interaction between the fibers and the medium is neglected, and the fibers are dimensionless.

To avoid these defaults, Briane [5,6] proposed two new models which are rigorously deduced from the homogenization of non-periodic fibrous microstructures. In the two models, the fibers are small radius cylinders periodically distributed in layers. Moreover, the fiber orientation is constant in each layer. In the first model (Sect. 1.2.1, Fig. 1), the layer width tends to zero but is large with respect to the fiber radius. In the second one (Sect. 1.2.1, Fig. 2), layers are replaced by rows whose width is of the same order as the fiber radius. So, the fiber orientation varies in a more realistic way in this second model. In both cases, the homogenization formula is far from being explicit since one needs to solve an auxiliary problem which is parametrized at each point of the domain (see Thm. 1.7). Therefore, it seems difficult to compare directly these models with the biomechanics one without additional hypothesis. Let us mention that another homogenization approach of the modeling of the myocardium was performed by Caillerie et al. in $[8,9]$. Their approach differs from Briane's one since they consider a large displacement framework and use a discrete homogenization process.

In this paper, our aim is to derive simplified homogenized models assuming small amplitude between the physical characteristics of the medium and the fibers. The small amplitude (or low contrast) homogenization theory was developed by Tartar [21,22] and, in particular, was applied by Allaire [1] and Allaire and Gutiérrez [2] in another context. First of all, we prove a new expansion formula (see Thm. 2.7) which extends to the anisotropic elasticity case the small amplitude homogenization formula obtained by Tartar [21] in the isotropic case. Then, we propose two models $(I$ and $I I)$ in conduction, which differ from each other by their geometry, and three in elasticity $(I, I I$ and $I I I)$. These models simplify the homogenized ones of $[5,6]$, taking into account the small amplitude assumption. Our approach allows us to validate or to refute the biomechanics heuristic model. Moreover, in some particular cases (model $I$ in conduction and model $I I I$ in elasticity) we obtain simple models of reinforcement by fibers of varying orientation. We only consider the linear case, both in conduction and in elasticity, although the nonlinear case seems more relevant for applications. Indeed, the biomechanics of the heart involves a large deformation approach, but this goes out of the present study (see the third point of Rem. 4.2). We restrict ourselves to a linear framework in order to focus on the non-periodic homogenization setting combined with the small amplitude assumption.

The three models are described in the sequel, where the parameter $\delta>0$ measures the low contrast between the fiber characteristics and the medium ones:

- In isotropic conduction, model $I$ in small amplitude gives (see Thm. 3.1) the following effective conductivity

$$
A_{\mathrm{eff}}^{I}=\alpha I_{3}+\beta(\tau \otimes \tau)+o\left(\delta^{2}\right)
$$

where $\tau$ is the fiber direction and $\alpha, \beta$ are explicit constants depending on $\delta$. Therefore, the biomechanics model coincides with this first homogenized model under the small amplitude assumption (neglecting the terms of order greater than 2).

- Model II (see Thm. 3.4) leads us to the following different homogenized conductivity

$$
A_{\mathrm{eff}}^{I I}=A_{\mathrm{eff}}^{I} \oplus D_{\mathrm{eff}}+o\left(\delta^{2}\right)
$$

The extra matrix $D_{\text {eff }}$ is zero where the fiber angle is constant. It is remarkable that the effective conductivity $A_{\text {eff }}^{I}$ of the first model is equal to the orthogonal projection of $A_{\text {eff }}^{I I}$ in the matrix space spanned by $I_{3}$ and $(\tau \otimes \tau)$. 
- In linear elasticity, model $I$ gives (see Thm. 4.1) the following effective stress matrix

$$
\begin{aligned}
\sigma_{\text {eff }}^{I}= & \mathbf{A}^{1} e+c_{1}\left(\mathbf{A}^{2} e-\mathbf{A}^{1} e\right)+\left[\mathbf{A}^{3} e+c_{2} \operatorname{tr}(e)(\tau \otimes \tau)+c_{3}(e \tau \cdot \tau) I_{3}+c_{4}(e(\tau \otimes \tau)+\tau \otimes e \tau)\right] \\
& +\delta^{2} \mathbf{N}^{I} e+o\left(\delta^{2}\right)
\end{aligned}
$$

where $\mathbf{A}^{1}, \mathbf{A}^{2}$ are, respectively, the Hooke's laws of the medium and of the fibers, $c_{i}$ are constants, $\mathbf{A}^{3}, \mathbf{N}^{I}$ are fourth order tensors and $\mathbf{A}^{3}$ is isotropic. Furthermore, models $I$ and $I I$ agree where the fiber angle is constant (see Thm. 4.3). Therefore, the biomechanics model does not coincide with the homogenized ones even under the small amplitude assumption.

- Due to the complexity of models $I$ and $I I$ in elasticity we consider model $I I I$ in which the fiber tensor $\mathbf{A}^{2}$ is deduced from the isotropic medium tensor $\mathbf{A}^{1}$ by a small anisotropic perturbation acting only in the fiber direction in the spirit of the biomechanics law (0.1), namely

$$
\mathbf{A}^{2} e:=\mathbf{A}^{1} e+\delta(e \tau \cdot \tau)(\tau \otimes \tau), \quad \text { with } \quad \mathbf{A}^{1} e:=\lambda_{1} \operatorname{tr}(e)+2 \mu_{1} e .
$$

Thanks to the anisotropic small amplitude formula of Theorem 2.7, we obtain (see Thm. 4.5) the following expansion

$$
\sigma_{\mathrm{eff}}^{I I I}=\mathbf{A}^{1} e+c\left(\mathbf{A}^{2} e-\mathbf{A}^{1} e\right)-\kappa_{\nu, \tau} \delta^{2} \frac{\mu_{1}+\lambda_{1}}{\mu_{1}\left(2 \mu_{1}+\lambda_{1}\right)}(e \tau \cdot \tau)(\tau \otimes \tau)+o\left(\delta^{2}\right)
$$

where $c$ and $\kappa_{\nu, \tau}$ are constants. Therefore, this model rigorously validates the biomechanics one at the second order, and provides a simple effective tensor.

The paper is organized as follows. In Section 1, we recall the notion of $H$-convergence and Briane homogenization results for the fibrous microstructures. In Section 2, we introduce the notion of $H$-measure and the small amplitude homogenization procedure due to Tartar. We conclude this section by a new small amplitude homogenization formula in anisotropic elasticity (Thm. 2.7). Section 3 is devoted to simplified models obtained in conduction under the small amplitude assumption. In Section 4 we derive the simplified models in linearized elasticity.

Along the paper, we will use the following basic notations:

\section{Notations}

- $N \in \mathbb{N}, N:=2$ or 3 in the Sections 3 and $4, N \geq 1$ in Sections 1 and 2.3.

- $Y_{N}$ is the cube $\left(-\frac{1}{2}, \frac{1}{2}\right)^{N}$ of $\mathbb{R}^{N}, N \geq 2$.

- For any subset $E$ of $\mathbb{R}^{N},|E|$ denotes the Lebesgue measure of $E$.

- $\mathcal{B}:=\left\{e_{1}, \ldots, e_{N}\right\}$ is the canonical basis of $\mathbb{R}^{N}$.

- If $x \in \mathbb{R}^{N}$, we denote by $x_{i}$ its coordinates: $x=\sum_{i=1}^{N} x_{i} e_{i}$.

- For $x, y \in \mathbb{R}^{N}, x \cdot y:=\sum_{i=1}^{N} x_{i} y_{i}$.

- We provide $\mathbb{R}^{N \times N}$ with the scalar product ":" defined by $A: B:=\operatorname{tr}\left(A^{T} B\right)$.

- For a parallelepiped $Z$ of $\mathbb{R}^{N}, H_{\#}^{1}(Z)$ (resp. $L_{\#}^{2}(Z)$ ) denotes the space of the $Z$-periodic functions which belong to $H_{\text {loc }}^{1}\left(\mathbb{R}^{N}\right)$ (resp. which belong to $L_{\text {loc }}^{2}\left(\mathbb{R}^{N}\right)$ ).

- For $f \in L_{\#}^{2}(Z),\langle f\rangle:=\frac{1}{|Z|} \int_{Z} f(x) \mathrm{d} x$ denotes the mean value. 


\section{Reviews of homogenization RESUlts}

\subsection{A few recalls of $H$-convergence}

\subsubsection{H-convergence in conduction}

We recall the definition and the "compactness theorem" of the $H$-convergence theory for second-order elliptic scalar equations introduced by Murat and Tartar [15] in the general case and by Spagnolo [18] (under the name of $G$-convergence) in the symmetric case.

Definition 1.1 (Murat and Tartar [15]). Let $\Omega$ be a bounded open set of $\mathbb{R}^{N}$.

(i) The space $\mathcal{M}(\alpha, \beta ; \Omega)$ is the set of matrix-valued functions $A: x \mapsto A(x)$ defined on $\Omega$ such that

$$
\forall \xi \in \mathbb{R}^{N}, \quad A(x) \xi \cdot \xi \geq \alpha|\xi|^{2} \quad \text { and } \quad A^{-1}(x) \xi \cdot \xi \geq \beta^{-1}|\xi|^{2} \quad \text { a.e. } x \in \Omega
$$

(ii) A sequence $A^{\varepsilon}$ of $\mathcal{M}(\alpha, \beta ; \Omega)$ is said to $H$-converge to $A_{\text {eff }}$ if $A_{\text {eff }} \in \mathcal{M}(\alpha, \beta ; \Omega)$ and if for any open set $\omega \Subset \Omega, f \in H^{-1}(\omega)$, the solution $u_{\varepsilon}$ of

$$
\left\{\begin{array}{l}
-\operatorname{div}\left(A_{\varepsilon} \nabla u_{\varepsilon}\right)=f \quad \text { in } \omega \\
u_{\varepsilon} \in H_{0}^{1}(\omega)
\end{array}\right.
$$

satisfies the weak convergences

$$
\left\{\begin{array}{rll}
u_{\varepsilon} & \longrightarrow u_{0} & H^{1}(\Omega) \text { weak } \\
A_{\varepsilon} \nabla u_{\varepsilon} & \longrightarrow A_{\mathrm{eff}} \nabla u_{0} & L^{2}\left(\Omega ; \mathbb{R}^{N}\right) \text { weak }
\end{array}\right.
$$

where $u_{0}$ is the solution of

$$
\left\{\begin{array}{l}
-\operatorname{div}\left(A_{\mathrm{eff}} \nabla u_{0}\right)=f \quad \text { in } \omega \\
u_{0} \in H_{0}^{1}(\omega)
\end{array}\right.
$$

The $H$-convergence of $A_{\varepsilon}$ to $A_{\text {eff }}$ is denoted by $A_{\varepsilon} \stackrel{H}{\longrightarrow} A_{\text {eff }}$.

The most important result of the $H$-convergence is the following "compactness theorem" due to Murat and Tartar:

Theorem 1.2 (Murat and Tartar [15]). If $A_{\varepsilon}$ is a sequence of $\mathcal{M}(\alpha, \beta ; \Omega)$ then there exists a subsequence, still denoted by $\varepsilon$, and $A_{\mathrm{eff}} \in \mathcal{M}(\alpha, \beta ; \Omega)$ such that $A_{\varepsilon} \stackrel{H}{\longrightarrow} A_{\mathrm{eff}}$.

\subsubsection{H-convergence in linearized elasticity}

We recall some basic definitions about elasticity and the definition of the $H$-convergence in linearized elasticity (see e.g. $[1,12]$ for a more complete presentation).

Let $\mathbb{R}_{s}^{N \times N}$ be the subset of $\mathbb{R}^{N \times N}$ of symmetric matrices and $\mathbf{M}_{N}^{4}$ be the set of symmetric fourth order tensors, i.e.

$$
\mathbf{M}_{N}^{4}:=\left\{\mathbf{A}:=\left(A_{i j k l}\right)_{1 \leq i, j, k, l \leq N} \mid A_{i j k l}=A_{k l i j}=A_{j i k l}=A_{i j l k}\right\} .
$$

For $u \in H^{1}\left(\Omega ; \mathbb{R}^{N}\right)$, we denote by $e(u)$ the strain matrix whose coefficients $e_{i j}(u)$ are given by

$$
e_{i j}(u)=\frac{1}{2}\left(\frac{\partial u_{i}}{\partial x_{j}}+\frac{\partial u_{j}}{\partial x_{i}}\right) .
$$

Definition 1.3. Let $\Omega$ be a bounded open set of $\mathbb{R}^{N}$. 
(i) We define the space $\mathcal{M}^{4}(\alpha, \beta ; \Omega)$ as the set of symmetric fourth order tensor valued functions $\mathbf{A}: x \rightarrow$ $\mathbf{A}(x)$ defined from $\Omega$ to $\mathbf{M}_{N}^{4}$ such that

$$
\forall e \in \mathbb{R}_{s}^{N \times N}, \quad \mathbf{A}(x) e: e \geq \alpha|e|^{2} \quad \text { and } \quad \mathbf{A}(x)^{-1} e: e \geq \beta^{-1}|e|^{2} \quad \text { a.e. } x \in \Omega .
$$

(ii) A sequence $\mathbf{A}_{\varepsilon}$ of $\mathcal{M}^{4}(\alpha, \beta ; \Omega)$ is said to $H$-converge to $\mathbf{A}_{\text {eff }}$ if $\mathbf{A}_{\text {eff }} \in \mathcal{M}^{4}(\alpha, \beta ; \Omega)$ and if for any open set $\omega \Subset \Omega, f \in H^{-1}\left(\omega ; \mathbb{R}^{N}\right)$, the solution $u_{\varepsilon}$ of the Dirichlet problem

$$
\left\{\begin{array}{l}
-\operatorname{div}\left(\mathbf{A}_{\varepsilon} e\left(u_{\varepsilon}\right)\right)=f \quad \text { in } \omega \\
u_{\varepsilon} \in H_{0}^{1}\left(\omega ; \mathbb{R}^{N}\right),
\end{array}\right.
$$

satisfies the weak convergences

$$
\left\{\begin{array}{cl}
u_{\varepsilon} \longrightarrow u_{0} & H_{0}^{1}\left(\Omega ; \mathbb{R}^{N}\right) \text { weak } \\
\mathbf{A}_{\varepsilon} e\left(u_{\varepsilon}\right) \longrightarrow \mathbf{A}_{\mathrm{eff}} e\left(u_{0}\right) & L^{2}\left(\Omega ; \mathbb{R}_{s}^{N \times N}\right) \text { weak }
\end{array}\right.
$$

where $u_{0}$ is the solution of

$$
\left\{\begin{array}{l}
-\operatorname{div}\left(\mathbf{A}_{\text {eff }}(x) e\left(u_{0}\right)\right)=f \quad \text { in } \omega, \\
u_{0} \in H_{0}^{1}\left(\omega ; \mathbb{R}^{N}\right) .
\end{array}\right.
$$

We denoted the $H$-convergence of $\mathbf{A}_{\varepsilon}$ to $\mathbf{A}_{\text {eff }}$ by $\mathbf{A}_{\varepsilon} \stackrel{H}{\longrightarrow} \mathbf{A}_{\text {eff }}$.

Remark 1.4. Theorem 1.2 established in the conduction case still holds true for the linearized elasticity case.

In the general case, there is no explicit formula for the homogenized law (neither in conduction and nor in linearized elasticity). Nevertheless, in the periodic case, i.e. $A_{\varepsilon}(x):=A\left(\frac{x}{\varepsilon}\right)$ p.p. $x \in \Omega$ where $A$ is periodic, the homogenized law can be explicitly computed (not totally explicit since one needs to solve a cell problem), see for example [4].

In the non-periodic case there is some particular microstructures for which the homogenized law can be explicitly obtained. For instance, in the next section we present two non-periodic microstructures due to Briane.

\subsection{The non-periodic fibrous microstructures}

In this section we briefly describe the geometries of the two fibrous microstructures studied in $[5,6]$ and we recall the homogenization results related to these microstructures.

\subsubsection{Geometries of the fibrous microstructures}

Let $\gamma \in C^{2}(\mathbb{R})$ with $|\gamma|<\frac{\pi}{2}$ and set

$$
\tau\left(x_{1}\right):=\cos \gamma\left(x_{1}\right) e_{2}+\sin \gamma\left(x_{1}\right) e_{3} .
$$

We consider the following two microstructures:

- First microstructure: we consider layers $\Omega_{\varepsilon}^{n}$ orthogonal to the $x_{1}$-axis of width $\varepsilon^{\alpha}$ where $0<\alpha<1$ in order to obtain layers of small width but large with respect to the fiber radius $\varepsilon r, r>0$ (see Fig. 1). In each layer we have a periodic lattice of fibers of period $\varepsilon$ with a constant orientation which depends only on the layer. Each fiber makes an angle $\gamma\left(x_{1}^{n}\right)$ with the $x_{2}$-axis in the layer $\Omega_{\varepsilon}^{n}$ where $x^{n}$ is any point of $\Omega_{\varepsilon}^{n}$.

We denote by $\chi_{\varepsilon}^{I}$ the characteristic function of this fiber lattice. 
- Second microstructure: in the first microstructure, the fibers have a locally constant orientation. In order to avoid this assumption, we consider rows orthogonal to the $x_{1}$-axis of width of order $\varepsilon$. In each row we have a periodic lattice of fibers of radius $\varepsilon r, r>0$ with a constant orientation which depends only on the row (see Fig. 2). Each fiber makes an angle $\gamma\left(x_{1}\right)$ with the $x_{2}$-axis where $x$ is any point of the fiber.

We denote by $\chi_{\varepsilon}^{I I}$ the characteristic function of this fiber lattice.

The difference between the two microstructures is that in the first one we consider layers and in the second one, the layers are replaced by rows. So, in the second microstructure, the fiber orientation varies in a more realistic way.

\subsubsection{H-convergence results for the fibrous microstructures}

Let $\chi_{C}^{\#}$ be the $Y_{3}$-periodic function defined on $Y_{3}$ as the characteristic function of the cylinder

$$
C:=\left\{x \in Y_{3} \mid x_{1}^{2}+x_{3}^{2} \leq r^{2}\right\}
$$

Let $a, b \in] 0,+\infty[$ and set

$$
B_{\varepsilon}(x):=B\left(\frac{x}{\varepsilon}\right), \quad \text { where } \quad B:=\left(a\left(1-\chi_{C}^{\#}\right)+b \chi_{C}^{\#}\right) I_{3} .
$$

Let $B_{\text {eff }}$ be the constant $H$-limit of $B_{\varepsilon}$ which is given (due to the symmetry) by the classical formula (see e.g. [4])

$$
\forall \xi \in \mathbb{R}^{3}, \quad B_{\text {eff }} \xi: \xi=\min \left\{f_{Y_{3}} B(y)(\xi+\nabla \varphi(y)) \cdot(\xi+\nabla \varphi(y)) \mathrm{d} y \mid \varphi \in H_{\#}^{1}\left(Y_{3}\right)\right\}
$$

We set

$$
A_{\varepsilon}^{I}:=\left(a\left(1-\chi_{\varepsilon}^{I}\right)+b \chi_{\varepsilon}^{I}\right) I_{3} .
$$

Then, one has the following homogenization result:

Theorem 1.5 (Briane $[5,6])$. The sequence $A_{\varepsilon}^{I} H$-converges to $A_{\mathrm{eff}}^{I}$ which satisfies

$$
A_{\text {eff }}^{I}(x)=R\left(x_{1}\right)^{T} B_{\text {eff }} R\left(x_{1}\right),
$$

where $B_{\text {eff }}$ is given by (1.3) and $R\left(x_{1}\right)$ is the orthogonal matrix defined by

$$
R\left(x_{1}\right):=\left(\begin{array}{ccc}
1 & 0 & 0 \\
0 & \cos \gamma\left(x_{1}\right) & \sin \gamma\left(x_{1}\right) \\
0 & -\sin \gamma\left(x_{1}\right) & \cos \gamma\left(x_{1}\right)
\end{array}\right) .
$$

Remark 1.6. In $[5,6]$, formula (1.4) was obtained using a locally periodic homogenization procedure. This is due to the fact that, in this microstructure, the number of fiber rows in each layer is very large.

Fix $z \in \mathbb{R}^{3}$ and let $\chi^{z}$ be the periodic characteristic function of the set composed of cylinders of radius $r$ parallel to the $x_{2}$-axis, the period of which is

$$
\left\{\begin{array}{l}
Y(z):=\left\{t_{1} e_{1}+t_{2} e_{2}+\left(t_{3}+t_{1} d(z)\right) e_{3} \mid 0 \leq t_{i} \leq 1,1 \leq i \leq 3\right\} \\
\text { where } d(z):=\gamma^{\prime}\left(z_{1}\right)\left(\cos \gamma\left(z_{1}\right) z_{2}+\sin \gamma\left(z_{1}\right) z_{3}\right)
\end{array}\right.
$$

represented in Figure 3.

We set

$$
B_{\varepsilon}^{z}(x):=B^{z}\left(\frac{x}{\varepsilon}\right), \quad \text { where } \quad B^{z}:=\left(a\left(1-\chi^{z}\right)+b \chi^{z}\right) I_{3} .
$$




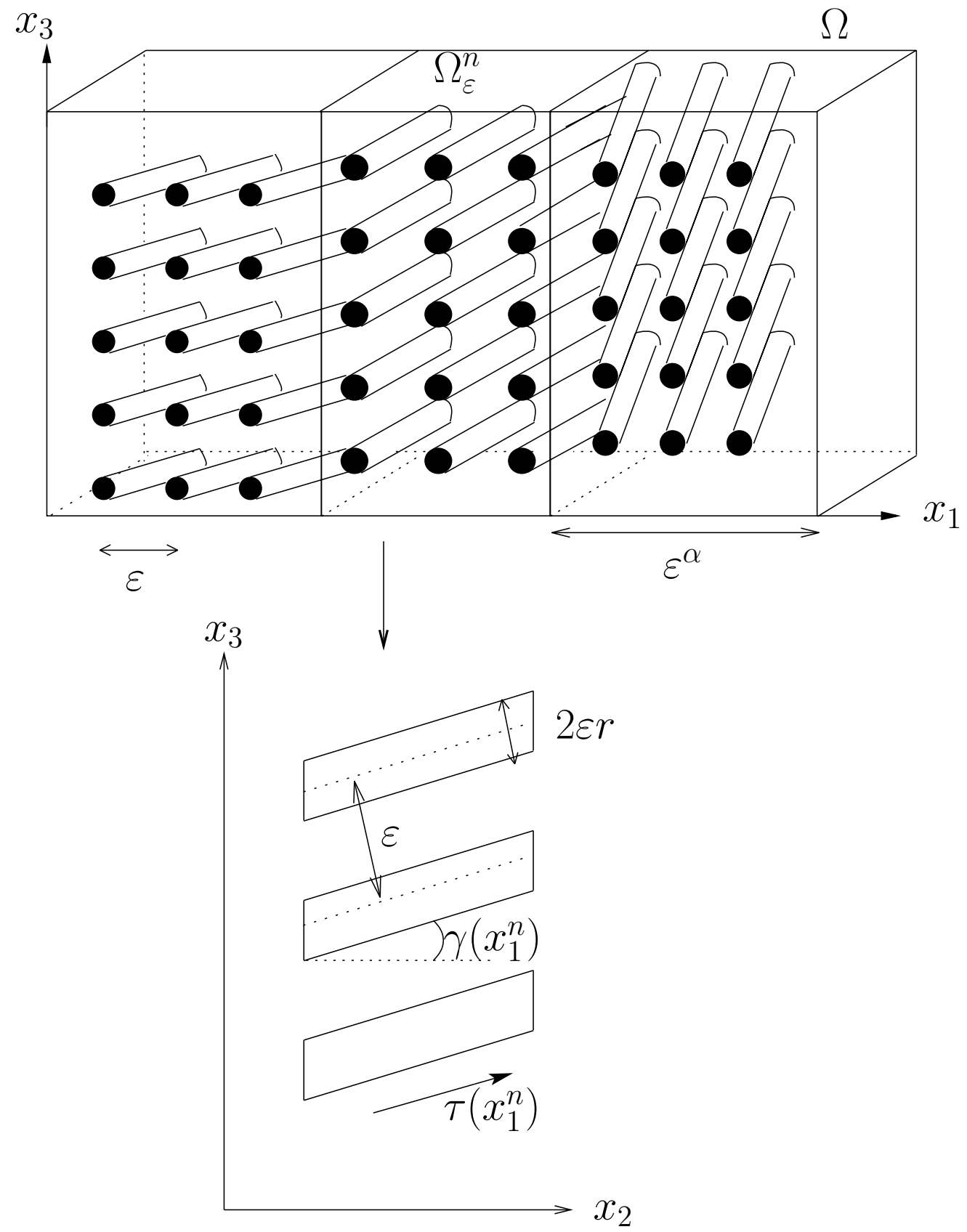

FigurE 1. Lattice of fibers of constant orientation by layer.

Let $B_{\text {eff }}^{z}$ be the constant $H$-limit of $B_{\varepsilon}^{z}$ which is given (due to the symmetry) by the classical formula (see e.g. [4])

$$
\forall \xi \in \mathbb{R}^{3}, \quad B_{\text {eff }}^{z} \xi: \xi=\min \left\{\int_{Y(z)} B^{z}(y)(\xi+\nabla \varphi(y)) \cdot(\xi+\nabla \varphi(y)) \mathrm{d} y \mid \varphi \in H_{\#}^{1}(Y(z))\right\}
$$




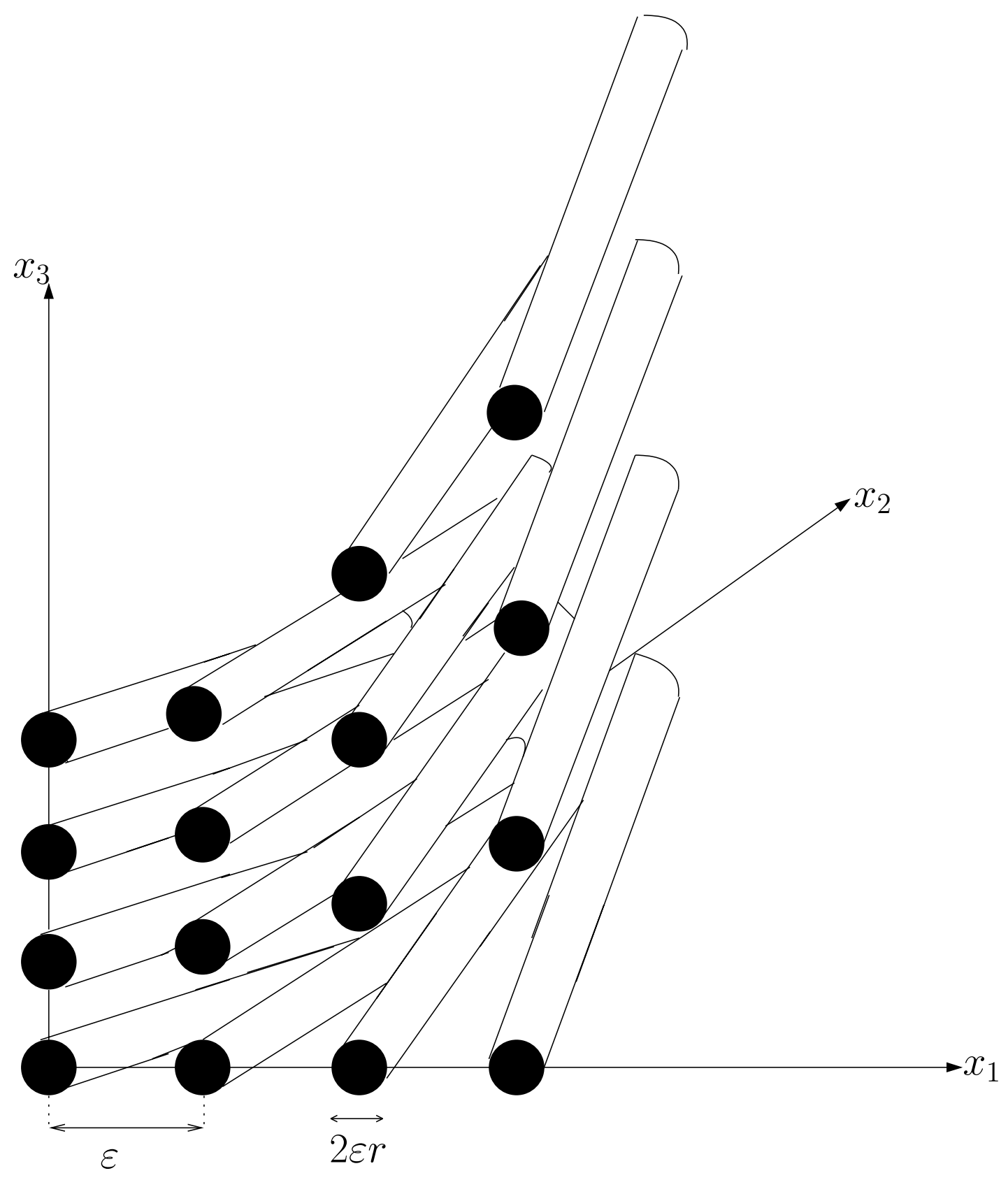

Figure 2. Lattice of fibers of constant orientation by row.

We set

$$
A_{\varepsilon}^{I I}:=\left(a\left(1-\chi_{\varepsilon}^{I I}\right)+b \chi_{\varepsilon}^{I I}\right) I_{3} .
$$

Then, one has the following homogenization result: 


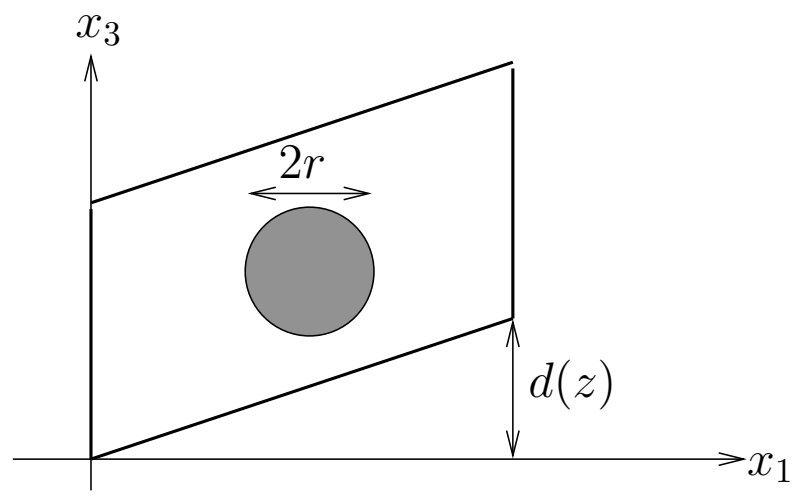

Figure 3. Section of $Y(z)$ in the plane $x_{1} O x_{3}$.

Theorem 1.7 (Briane [5-7]). The sequence $A_{\varepsilon}^{I I} H$-converges to the matrix-valued function $A_{\mathrm{eff}}^{I I}$ where, for any fixed $x, A_{\mathrm{eff}}^{I I}(x)$ is given by

$$
A_{\mathrm{eff}}^{I I}(x)=R\left(x_{1}\right)^{T} B_{\mathrm{eff}}^{x} R\left(x_{1}\right),
$$

with $B_{\mathrm{eff}}^{x}$ given by $(1.7)$ and $R\left(x_{1}\right)$ the rotation matrix defined by (1.5).

Remark 1.8. Contrary to the first one, the second microstructure is no more locally periodic. Theorem 1.7 was obtained by an approximation of the microstructure on each point $z$ by a locally periodic material parametrized by $z$. The fiber lattice which approximates the one of Figure 2 is periodic up to a meso scale $\varepsilon^{s}$ with $0<s<1$. It can be regarded as the tangent lattice of the original one. Its construction is then linear and based on a first-order Taylor expansion of the angle $\gamma$; it follows the appearance of the derivative of $\gamma$ in the period cell $Y(z)$.

\section{2. $H$-MEASURES AND SMALL AMPLITUde HOMOGENIZATION}

\subsection{Reviews on $H$-measures and small amplitude homogenization}

The notion of $H$-measure has been developed independently by Gérard [13] and Tartar [22]. Here, we will consider its application to explicit formulas in small amplitude homogenization introduced by Tartar. We recall the definition of the $H$-measures, the expression of the $H$-measure of a periodic function and the small amplitude homogenization formula in conduction. We refer to [22] for the proof of these results.

For all subset $\Omega$ of $\mathbb{R}^{N}$, we denote by $C(\Omega)$ the space of continuous real-valued functions on $\Omega$ and $C_{c}(\Omega)$ the subspace of $C(\Omega)$ formed of functions with compact support. Furthermore, we denote by $C_{0}\left(\mathbb{R}^{N}\right)$ the space of continuous complex-valued functions decreasing to 0 at infinity. We define the Fourier transform $\mathcal{F}$ on the space of rapidly decreasing functions $\mathcal{S}\left(\mathbb{R}^{N}\right)$ by

$$
\forall u \in \mathcal{S}\left(\mathbb{R}^{N}\right), \quad \mathcal{F} u(\xi):=\int_{\mathbb{R}^{N}} u(x) \mathrm{e}^{-2 \mathrm{i} \pi x \cdot \xi} \mathrm{d} x .
$$

Theorem 2.1 (Tartar [22]. Existence of $H$-measures). Let $U^{\varepsilon}$ be a sequence converging weakly to 0 in $L^{2}\left(\mathbb{R}^{N} ; \mathbb{R}^{p}\right)$. Then, up to a subsequence, there exists a family $\mu_{i, j}, i, j \in\{1, \ldots, p\}$, of complex-valued Radon measures on $\mathbb{R}^{N} \times S^{N-1}$ such that for every $\phi_{1}, \phi_{2} \in C_{0}\left(\mathbb{R}^{N}\right)$ and for all $\psi \in C\left(S^{N-1}\right)$, we have

$$
\left\langle\mu_{i, j}, \phi_{1} \overline{\phi_{2}} \otimes \psi\right\rangle=\lim _{\varepsilon \rightarrow 0} \int_{\mathbb{R}^{N}}\left[\mathcal{F}\left(\phi_{1} U_{i}^{\varepsilon}\right)(\xi)\right] \overline{\left[\mathcal{F}\left(\phi_{2} U_{j}^{\varepsilon}\right)(\xi)\right]} \psi\left(\frac{\xi}{|\xi|}\right) \mathrm{d} \xi .
$$


The matrix-valued measure $\mu$ of coefficients $\mu_{i, j}$ given by (2.1) is called the $H$-measure associated with the extracted subsequence of $U^{\varepsilon}$.

\section{Remark 2.2.}

1. Taking into account oscillation directions, the $H$-measure quantifies the lack of compactness of weakly converging sequences. This is due to the fact that, taking $\psi \equiv 1$ (see Cor. 1.4 of [22]), the sequence $U_{i}^{\varepsilon} U_{j}^{\varepsilon}$ weakly converges in the distribution sense to the measure $\nu$ given by

$$
\forall \phi \in C_{0}\left(\mathbb{R}^{N}\right), \quad\langle\nu, \phi\rangle:=\left\langle\mu_{i, j}, \phi \otimes 1\right\rangle
$$

2. In equality (2.1), the right hand-side only depends on the product $\phi_{1} \bar{\phi}_{2}$ instead of the couple $\left(\phi_{1}, \phi_{2}\right)$ (see [22] for details).

3. The $H$-measures are hermitian, i.e. $\bar{\mu}_{i, j}=\mu_{j, i} \quad \forall i, j \in\{1, \ldots, p\}$.

Let $u \in L_{\#}^{2}\left(Y_{N}\right)$, denote by $\langle u\rangle$ the mean value of $u$ on $Y_{N}$ and set $u_{\varepsilon}(x):=u\left(\frac{x}{\varepsilon}\right)$. Then the sequence $u_{\varepsilon}-\langle u\rangle$ converges to zero in $L^{2}\left(\mathbb{R}^{N}\right)$ weak. A formula for the $H$-measure associated with the sequence $u_{\varepsilon}-\langle u\rangle$ was obtained in [22] (Ex. 2.1). The proof of this result can be easily generalized to the case of any period.

Let $Y_{f}$ be the parallelepiped of $\mathbb{R}^{N}$ defined by

$$
Y_{f}:=\left\{\sum_{i=1}^{N} \lambda_{i} f_{i} \mid-\frac{1}{2} \leq \lambda_{i} \leq \frac{1}{2}, \forall i=1, \ldots, N\right\}
$$

where $\mathcal{B}^{\prime}:=\left\{f_{1}, \ldots, f_{N}\right\}$ is a basis of $\mathbb{R}^{N}$. Let $P$ be the matrix of $\mathbb{R}^{N}$ such that $P \mathcal{B}=\mathcal{B}^{\prime}$, where $\mathcal{B}$ is the canonical basis of $\mathbb{R}^{N}$. In the general case of a periodic function of period $Y_{f}$ we have the following result:

Proposition 2.3. Let $\Omega$ be a smooth bounded open set (with Lipschitz boundary) of $\mathbb{R}^{N}$. Let $u \in L_{\#}^{2}\left(Y_{f}\right)$. We set $u_{\varepsilon}(x):=u\left(\frac{x}{\varepsilon}\right)$ and we denote by $\mu$ the $H$-measure associated with the sequence $\left(u_{\varepsilon}-\langle u\rangle\right)$. Then, we have

$$
\mu=\sum_{\omega \in \mathbb{Z}_{f}^{3} \backslash\{0\}}\left|\hat{u}_{\omega}\right|^{2} \otimes \delta_{\frac{\omega}{|\omega|}} \quad \text { in } \Omega \times S^{N-1},
$$

where

$$
\hat{u}_{\omega}=\frac{1}{\left|Y_{f}\right|} \int_{Y_{f}} u(y) \mathrm{e}^{-2 i \pi \omega \cdot y} \mathrm{~d} y \quad \text { and } \quad \mathbb{Z}_{f}^{3}:=\left\{\left(P^{-1}\right)^{T} k \mid k \in \mathbb{Z}^{N}\right\} .
$$

Now, we recall the small amplitude homogenization formula in conduction.

Let $\Omega$ be an open set of $\mathbb{R}^{N}$ and $A_{0}, B_{\varepsilon}, C_{\varepsilon} \in L^{\infty}\left(\Omega ; \mathbb{R}^{N \times N}\right)$. We assume:

(i) $A_{0}$ is continuous and equi-coercive, i.e. $\exists \alpha>0, \forall \xi \in \mathbb{R}^{N}, \quad A_{0}(x) \xi \cdot \xi \geq \alpha|\xi|^{2} \quad$ a.e. $x \in \Omega$,

(ii) $B_{\varepsilon} \longrightarrow B_{0} \quad L^{\infty}\left(\Omega ; \mathbb{R}^{N \times N}\right)$ weak*,

(iii) $C_{\varepsilon} \longrightarrow C_{0} \quad L^{\infty}\left(\Omega ; \mathbb{R}^{N \times N}\right)$ weak*.

Let $\mu$ be the $H$-measure associated with the sequence $\left(B_{\varepsilon}-B_{0}\right) ; \mu=\left(\mu_{i j, k l}\right)_{i, j, k, l \in\{1, \ldots, N\}}$. We set

$$
A_{\varepsilon}(x ; \delta):=A_{0}(x)+\delta B_{\varepsilon}(x)+\delta^{2} C_{\varepsilon}(x)
$$

Theorem 2.4 (Tartar [22]). There exists a subsequence, still denoted by $\varepsilon$, such that for all $\delta>0$ small enough, we have

$$
A_{\varepsilon}(\cdot, \delta) \stackrel{H}{\longrightarrow} A_{\mathrm{eff}}(\cdot, \delta) \text { on } \Omega
$$

with

$$
A_{\mathrm{eff}}(x ; \delta)=A_{0}(x)+\delta B_{0}(x)+\delta^{2}\left(C_{0}(x)-M(x)\right)+o\left(\delta^{2}\right)
$$


where the matrix-valued measure $M$, called H-correction, associated with the expansion of the effective matrix has its coefficients given by

$$
\forall \phi \in C_{c}(\Omega), \quad \int_{\Omega} M_{i j}(x) \phi(x) \mathrm{d} x:=\sum_{k, l=1}^{N}\left\langle\mu_{i k, l j}, \frac{\phi(x) \xi_{k} \xi_{l}}{A_{0}(x) \xi \cdot \xi}\right\rangle,
$$

for any $i, j \in\{1, \ldots, N\}$.

Notation 2.5. Let $\mu$ be an $H$-measure and $\psi \in C\left(S^{N-1}\right), \varphi \in C(\Omega)$, then we define a measure denoted by $\psi(\xi) \varphi(x) \nu$ on $\Omega$ by

$$
\forall \phi \in C_{c}(\Omega), \quad\langle\psi(\xi) \varphi(x) \nu, \phi\rangle:=\langle\mu, \phi \varphi \otimes \psi\rangle .
$$

If the measure $\psi(\xi) \varphi(x) \nu$ admits a density with respect to the Lebesgue measure, the density will also be denoted by $\psi(\xi) \varphi(x) \nu$.

Example 2.6. Consider $A_{\varepsilon}$ satisfying the conditions of Theorem 2.4. By the previous notation, the $H$ correction $M$ is given by

$$
M_{i j}(x)=\sum_{k, l=1}^{N} \frac{\xi_{k} \xi_{l}}{A_{0}(x) \xi \cdot \xi} \mu_{i k, l j} \quad i, j \in\{1, \ldots, N\} .
$$

Furthermore, if $A_{0}:=a I_{N}$, since the $H$-measure has its support included in $\mathbb{R}^{N} \times S^{N-1}$, we obtain for any $i, j \in\{1, \ldots, N\}$

$$
M_{i j}(x)=\sum_{k, l=1}^{N} \frac{\xi_{k} \xi_{l}}{a(x)|\xi|^{2}} \mu_{i k, l j}=\sum_{k, l=1}^{N} \frac{\xi_{k} \xi_{l}}{a(x)} \mu_{i k, l j}
$$

\subsection{Small amplitude homogenization in elasticity}

We give a new small amplitude homogenization formula which extends to anisotropic elasticity the one of Tartar [21]. Here, we only assume the isotropy of the zero-order term in the expansion of $\mathbf{A}_{\varepsilon}$.

Let $\Omega$ be an open set of $\mathbb{R}^{N}$ and $\mathbf{A}_{0}, \mathbf{B}_{\varepsilon}, \mathbf{C}_{\varepsilon} \in L^{\infty}\left(\Omega ; \mathbf{M}_{N}^{4}\right)$. We assume:

(i) $\mathbf{A}_{0}$ is continuous and equi-coercive i.e. $\exists \alpha>0, \forall e \in \mathbb{R}_{s}^{N \times N}, \quad \mathbf{A}_{0}(x) e: e \geq \alpha|e|^{2} \quad$ a.e. $x \in \Omega$,

(ii) $\mathbf{B}_{\varepsilon} \longrightarrow \mathbf{B}_{0} L^{\infty}\left(\Omega ; \mathbf{M}_{N}^{4}\right)$ weak*,

(iii) $\mathbf{C}_{\varepsilon} \longrightarrow \mathbf{C}_{0} \quad L^{\infty}\left(\Omega ; \mathbf{M}_{N}^{4}\right)$ weak*.

Let $\mu$ be the $H$-measure associated with the sequence $\left(\mathbf{B}_{\varepsilon}-\mathbf{B}_{0}\right) ; \mu=\left(\mu_{i j k l, m n p q}\right)_{i, j, k, l, m, n, p, q \in\{1, \ldots, N\}}$. We set

$$
\mathbf{A}_{\varepsilon}(x ; \delta):=\mathbf{A}_{0}(x)+\delta \mathbf{B}_{\varepsilon}(x)+\delta^{2} \mathbf{C}_{\varepsilon}(x)
$$

The new small amplitude formula is given by the following result:

Theorem 2.7. Assume that $\mathbf{A}_{0}$ is isotropic and denote by $\lambda_{0}$ and $\mu_{0}$ the Lamé coefficients of $\mathbf{A}_{0}$ (which are continuous functions on $\Omega$ ). Then there exists a subsequence, still denoted by $\varepsilon$, such that for all $\delta>0$ small enough, we have

$$
\mathbf{A}_{\varepsilon}(\cdot, \delta) \stackrel{H}{\longrightarrow} \mathbf{A}_{\mathrm{eff}}(\cdot, \delta) \text { on } \Omega,
$$

with

$$
\mathbf{A}_{\mathrm{eff}}(x ; \delta)=\mathbf{A}_{0}(x)+\delta \mathbf{B}_{0}(x)+\delta^{2}\left(\mathbf{C}_{0}(x)-\mathbf{M}(x)\right)+o\left(\delta^{2}\right),
$$


where the fourth-order tensor valued measure $\mathbf{M}$, called H-correction, associated with the expansion of the effective tensor has its coefficients given by

$$
\begin{aligned}
\forall \phi \in C_{c}(\Omega), \int_{\Omega} M_{i j k l}(x) \phi(x) \mathrm{d} x & =\sum_{m, p, q=1}^{N}\left\langle\mu_{i j p q, q m k l}, \frac{\xi_{m} \xi_{p} \phi}{\mu_{0}}\right\rangle \\
& -\sum_{m, n, p, q=1}^{N}\left\langle\mu_{i j p q, m n k l}, \frac{\mu_{0}+\lambda_{0}}{\mu_{0}\left(2 \mu_{0}+\lambda_{0}\right)} \xi_{m} \xi_{n} \xi_{p} \xi_{q} \phi\right\rangle,
\end{aligned}
$$

for any $i, j, k, l \in\{1, \ldots, N\}$.

Remark 2.8. In the case of an isotropic Hooke's law $\mathbf{A}_{\varepsilon}$ with expansion of the type (2.5), the Lamé coefficients $\mu^{\varepsilon}$ and $\lambda^{\varepsilon}$ of $\mathbf{A}_{\varepsilon}$ read as

$$
\mu^{\varepsilon}=\mu_{0}+\mu_{1}^{\varepsilon} \delta+\mu_{2}^{\varepsilon} \delta^{2} \quad \text { and } \quad \lambda^{\varepsilon}=\lambda_{0}+\lambda_{1}^{\varepsilon} \delta+\lambda_{2}^{\varepsilon} \delta^{2}
$$

where

$$
\mu_{i}^{\varepsilon} \longrightarrow \mu_{i} L^{\infty}(\Omega) \text { weak }^{*} \text { and } \lambda_{i}^{\varepsilon} \longrightarrow \lambda_{i} \quad L^{\infty}(\Omega) \text { weak }^{*} \text { for } i=1,2 .
$$

Let $\nu$ be the $H$-measure associated with the sequence $\left(\mu_{1}^{\varepsilon}-\mu_{1}, \lambda_{1}^{\varepsilon}-\lambda_{1}\right)$. We set (with Notation 2.5):

$$
a_{i j}:=\xi_{i} \xi_{j} \nu_{11}, \quad b_{i j}:=\xi_{i} \xi_{j} \operatorname{Re}\left(\nu_{12}\right) \quad \text { and } \quad a_{i j k l}:=\xi_{i} \xi_{j} \xi_{k} \xi_{l} \nu_{11}, \quad i, j, k, l \in\{1, \ldots, N\}
$$

We also note $\operatorname{tr}\left(\nu_{22}\right)$ the measure defined on $\Omega$ by

$$
\forall \phi \in C_{c}(\Omega),\left\langle\operatorname{tr}\left(\nu_{22}\right), \phi\right\rangle:=\left\langle\nu_{22}, \phi \otimes 1\right\rangle
$$

Then formula (2.6) reads as, for all $i, j, k, l \in\{1, \ldots, N\}$,

$$
\begin{aligned}
M_{i j k l}= & \frac{1}{\mu_{0}}\left(\delta_{j k} a_{i l}+\delta_{i k} a_{j l}+\delta_{j l} a_{i k}+\delta_{i l} a_{j k}\right)-\frac{4\left(\mu_{0}+\lambda_{0}\right)}{\mu_{0}\left(2 \mu_{0}+\lambda_{0}\right)} a_{i j k l} \\
& +\frac{2}{2 \mu_{0}+\lambda_{0}}\left(\delta_{k l} b_{i j}+\delta_{i j} b_{k l}\right)+\frac{1}{2 \mu_{0}+\lambda_{0}} \delta_{i j} \delta_{k l} \operatorname{tr}\left(\nu_{22}\right)
\end{aligned}
$$

which is the formula established in [21] and also in [1] for the periodic case. See Section 2.3.2 for the proof of $(2.7)$.

\subsection{Proofs in the anisotropic case}

\subsubsection{Proof of Theorem 2.7}

We follow the same procedure as Tartar [22]. Here, the difficulty comes from delicate algebraic computations due to the anisotropy of $\mathbf{A}_{\varepsilon}$.

By Proposition 17 of [23], we have that there exists a subsequence, still denoted by $\varepsilon$, such that, for any $\delta$ small enough, $\mathbf{A}_{\varepsilon}(\cdot, \delta) H$-converges to $\mathbf{A}_{\text {eff }}(\cdot, \delta)$, where $\mathbf{A}_{\text {eff }}(\cdot, \delta)$ is analytic in $\delta$.

Remark 2.9. For any $\mathbf{A} \in \mathbf{M}_{N}^{4}$ and $u \in H^{1}\left(\Omega ; \mathbb{R}^{N}\right)$, we have

$$
\mathbf{A} e(u)=\mathbf{A} \nabla u, \quad \text { where } \quad(\nabla u)_{i j}=\frac{\partial u_{j}}{\partial x_{i}} \quad i, j \in\{1, \ldots, N\},
$$

since $\mathbf{A}$ is symmetric. Thus, in Definition 1.3 we can replace $e\left(u_{\varepsilon}\right)$ by $\nabla u_{\varepsilon}$. 
Let $u \in H_{0}^{1}\left(\Omega ; \mathbb{R}^{N}\right), \omega \Subset \Omega$ and $u_{\varepsilon}^{\delta}$ be the solution of

$$
\left\{\begin{array}{l}
-\operatorname{div}\left(\mathbf{A}_{\varepsilon}(x, \delta) \nabla u_{\varepsilon}^{\delta}\right)=-\operatorname{div}\left(\mathbf{A}_{\mathrm{eff}}(x, \delta) \nabla u\right) \quad \text { in } \omega, \\
u_{\varepsilon}^{\delta} \in H_{0}^{1}\left(\omega ; \mathbb{R}^{N}\right) .
\end{array}\right.
$$

As usual in $H$-convergence theory, it is enough to compute $\mathbf{A}_{\text {eff }} \nabla u$ in $\omega$ to obtain $\mathbf{A}_{\text {eff }}$ on $\Omega$. Furthermore, by definition of the $H$-convergence, $\mathbf{A}_{\text {eff }} \nabla u$ is obtained as the limit in $L^{2}\left(\omega ; \mathbb{R}^{N}\right)$ weak of $\mathbf{A}_{\varepsilon} \nabla u_{\varepsilon}^{\delta}$.

Preliminary. By Definition 1.3 of the $H$-convergence, we have

$$
\left\{\begin{array}{cl}
u_{\varepsilon}^{\delta} \longrightarrow u & H^{1}\left(\omega ; \mathbb{R}^{N}\right) \text { weak } \\
\mathbf{A}_{\varepsilon}(., \delta) \nabla u_{\varepsilon}^{\delta} \longrightarrow \mathbf{A}_{\text {eff }}(., \delta) \nabla u & L^{2}\left(\omega ; \mathbb{R}^{N \times N}\right) \text { weak }
\end{array}\right.
$$

Let $\varphi \in C_{c}^{\infty}(\Omega)$ such that $\varphi \equiv 1$ on $\omega$, and define

$$
E_{\varepsilon}(x, \delta):=\nabla\left(\varphi u_{\varepsilon}^{\delta}\right) \quad \text { and } \quad D_{\varepsilon}(x, \delta):=\mathbf{A}_{\varepsilon}(x, \delta) E_{\varepsilon}(x, \delta) .
$$

We have

for any $1 \leq i, j, k \leq N$. Moreover

$$
\left\{\begin{aligned}
\partial_{k}\left(E_{\varepsilon}\right)_{i, j} & =\partial_{i}\left(E_{\varepsilon}\right)_{k, j} & & \text { in } H^{-1}\left(\mathbb{R}^{N}\right) \\
E_{\varepsilon} & \longrightarrow \nabla u & & L^{2}\left(\omega ; \mathbb{R}^{N \times N}\right) \text { weak }
\end{aligned}\right.
$$

$$
\left\{\begin{array}{cl}
\operatorname{div}\left(D_{\varepsilon}\right)=\operatorname{div}\left(\mathbf{A}_{\mathrm{eff}} \nabla u\right) & \text { in } \omega, \\
D_{\varepsilon} \longrightarrow \mathbf{A}_{\mathrm{eff}} \nabla u & L^{2}\left(\omega ; \mathbb{R}^{N \times N}\right) \text { weak. }
\end{array}\right.
$$

The functions $E_{\varepsilon}$ and $D_{\varepsilon}$ are analytic in $\delta$. From (2.5), combined with (2.8) and considering the case $\delta=0$, we obtain the following asymptotic expansions

$$
E_{\varepsilon}(x, \delta)=\nabla u(x)+\delta E_{\varepsilon}^{1}(x)+\delta^{2} E_{\varepsilon}^{2}(x)+o\left(\delta^{2}\right),
$$

where $E_{\varepsilon}^{1}, E_{\varepsilon}^{2} \longrightarrow 0$ in $L^{2}\left(\Omega ; \mathbb{R}^{N \times N}\right)$ weak, and

$$
\begin{aligned}
D_{\varepsilon}(x ; \delta)= & \mathbf{A}_{0}(x) \nabla u(x)+\delta\left(\mathbf{A}_{0}(x) E_{\varepsilon}^{1}(x)+\mathbf{B}_{\varepsilon} \nabla u(x)\right) \\
& +\delta^{2}\left(\mathbf{A}_{0}(x) E_{\varepsilon}^{2}(x)+\mathbf{B}_{\varepsilon}(x) E_{\varepsilon}^{1}(x)+\mathbf{C}_{\varepsilon}(x) \nabla u(x)\right)+o\left(\delta^{2}\right) .
\end{aligned}
$$

Since $\mathbf{A}_{\text {eff }}$ is analytic in $\delta$, it admits an expansion of the type

$$
\mathbf{A}_{\text {eff }}(x, \delta)=\mathbf{A}^{*}(x)+\mathbf{B}^{*}(x) \delta+\mathbf{C}^{*}(x) \delta^{2}+o\left(\delta^{2}\right) .
$$

Then, if we denote by $\lim _{L^{2}-w}$ the weak limit in $L^{2}\left(\omega ; \mathbb{R}^{N \times N}\right)$, from (2.10) and (2.12) we obtain

$$
\begin{aligned}
& \mathbf{A}^{*} \nabla u=\mathbf{A}_{0} \nabla u \\
& \mathbf{B}^{*} \nabla u=\lim _{L^{2}-w}\left(\mathbf{A}_{0} E_{\varepsilon}^{1}+\mathbf{B}_{\varepsilon} \nabla u\right)=\mathbf{B}_{0} \nabla u \\
& \mathbf{C}^{*} \nabla u=\lim _{L^{2}-w}\left(\mathbf{A}_{0} E_{\varepsilon}^{2}+\mathbf{B}_{\varepsilon} E_{\varepsilon}^{1}+\mathbf{C}_{\varepsilon} \nabla u\right)=\lim _{L^{2}-w}\left(\mathbf{B}_{\varepsilon}-\mathbf{B}_{0}\right) E_{\varepsilon}^{1}+\mathbf{C}_{0} \nabla u
\end{aligned}
$$

since $\lim _{L^{2}-w} \mathbf{B}_{0} E_{\varepsilon}^{1}=\lim _{L^{2}-w} \mathbf{A}_{0} E_{\varepsilon}^{2}=0$.

It remains to compute the weak limit of $\left(\mathbf{B}_{\varepsilon}-\mathbf{B}_{0}\right) E_{\varepsilon}^{1}$. In order to compute this limit we proceed in three steps. 
1. In the first step, we compute the $L^{2}\left(\omega ; \mathbb{R}^{N \times N}\right)$ weak limit of $\left(\mathbf{B}_{\varepsilon}-\mathbf{B}_{0}\right) E_{\varepsilon}^{1}$ in terms of the $H$-measure $\mu^{\prime}$ associated with the tensor-valued function $\mathbf{K}_{\varepsilon}:=\left(\mathbf{B}_{\varepsilon}-\mathbf{B}_{0}, E_{\varepsilon}^{1}\right)\left(\mathbf{K}_{\varepsilon}\right.$ has for coefficients $\left(K_{\varepsilon}\right)_{i j k l}$, with $1 \leq i, j \leq N$ and $1 \leq k, l \leq N+1$, see (2.18)).

2. In the second step, from Theorem 1.6 of [22], we express the sum $\sum_{p, q} \mu_{i j p q, p q(N+1)(N+1)}^{\prime}$ for $m, n, p, q \in$ $\{1, \ldots, N\}$ in terms of the $H$-measure $\mu$ associated with $\left(\mathbf{B}_{\varepsilon}-\mathbf{B}_{0}\right)$ using three algebraic computations.

3. Combining the results of steps 1 and 2 we determine the $L^{2}\left(\omega ; \mathbb{R}^{N \times N}\right)$ weak limit of $\left(\mathbf{B}_{\varepsilon}-\mathbf{B}_{0}\right) E_{\varepsilon}^{1}$ in terms of the $H$-measure $\mu$ and we obtain the formula for $\mathbf{A}_{\text {eff }}$.

First step. From (2.10), (2.12) and (2.15), we have

$$
\operatorname{div}\left(\mathbf{A}_{0} E_{\varepsilon}^{1}+\left(\mathbf{B}_{\varepsilon}-\mathbf{B}_{0}\right) \nabla u\right)=0 \quad \text { in } H^{-1}(\omega) .
$$

In the sequel, we choose $u$ such that $\nabla u$ is continuous. Denote by $\mathbf{K}_{\varepsilon}:=\left(\mathbf{B}_{\varepsilon}-\mathbf{B}_{0}, E_{\varepsilon}^{1}\right)$ the tensor-valued function defined on $\omega$ by

$$
\left(K_{\varepsilon}\right)_{i j k l}:= \begin{cases}\left(B_{\varepsilon}-B_{0}\right)_{i j k l} & \text { if } 1 \leq i, j, k, l \leq N, \\ \left(E_{\varepsilon}^{1}\right)_{i j} & \text { if } k=l=N+1 .\end{cases}
$$

Then

$$
\left(\left(\mathbf{B}_{\varepsilon}-\mathbf{B}_{0}\right) E_{\varepsilon}^{1}\right)_{i j}=\sum_{k, l=1}^{N}\left(K_{\varepsilon}\right)_{i j k l}\left(K_{\varepsilon}\right)_{k l(N+1)(N+1)} .
$$

We denote by $\mu^{\prime}$ the $H$-measure associated with $\mathbf{K}_{\varepsilon}$ (the coefficients of $\mu^{\prime}$ are the measures $\mu_{i j k l, m n p q}^{\prime}$ with $1 \leq i, j, m, n \leq N$ and $1 \leq k, l, p, q \leq N+1$ ). We have from (2.19), thanks to Corollary 1.4 of [22], for all $\phi \in C_{c}(\omega)$,

$$
\int_{\mathbb{R}^{N}}\left(\left(\mathbf{B}_{\varepsilon}-\mathbf{B}_{0}\right)(x) E_{\varepsilon}^{1}(x)\right)_{i j} \phi(x) \mathrm{d} x \underset{\varepsilon \rightarrow 0}{\longrightarrow} \sum_{k, l=1}^{N}\left\langle\mu_{i j k l, k l(N+1)(N+1)}^{\prime}, \phi \otimes 1\right\rangle .
$$

Note that $\mu_{i j k l, m n p q}^{\prime}=\mu_{i j k l, m n p q}$, for all $i, j, k, l, m, n, p, q \in\{1, \ldots, N\}$. Therefore, to obtain the $L^{2}\left(\omega ; \mathbb{R}^{N \times N}\right)$ weak limit of $\left(\mathbf{B}_{\varepsilon}-\mathbf{B}_{0}\right) E_{\varepsilon}^{1}$ in terms of the $H$-measure $\mu$, it is enough to express the right hand-side of (2.20) in term of $\mu_{i j k l, m n p q}^{\prime}$, with $i, j, k, l, m, n, p, q \in\{1, \ldots, N\}$. This is the goal of the next step.

Second step. We express the right hand-side of (2.20) in terms of the $H$-measure $\mu$ by using Theorem 1.6 (localisation principle) of [22] and algebraic computations. Since the coefficients $\left(A_{0}\right)_{i j k l}, \partial_{k} u_{l}$ are continuous in $\Omega$, by the localisation principle, (2.17) yields

$$
\sum_{j, k, l=1}^{N} \xi_{j}\left(\left(A_{0}\right)_{i j k l}(x) \mu_{k l(N+1)(N+1), m n p q}^{\prime}+\partial_{k} u_{l}(x) \mu_{i j k l, m n p q}^{\prime}\right)=0 \quad \text { in } \omega \times S^{N-1},
$$

for all $p, q \in\{1, \ldots, N+1\}$ and $i, m, n \in\{1, \ldots, N\}$. The isotropy of $\mathbf{A}_{0}$ gives

$$
\left(A_{0}\right)_{i j k l}=\lambda_{0} \delta_{i j} \delta_{k l}+\mu_{0}\left(\delta_{i k} \delta_{j l}+\delta_{i l} \delta_{j k}\right),
$$

which implies

$$
\begin{aligned}
\mu_{0}(x)\left(\sum_{j=1}^{N} \xi_{j} \mu_{i j(N+1)(N+1), m n p q}^{\prime}+\sum_{j=1}^{N} \xi_{j} \mu_{j i(N+1)(N+1), m n p q}^{\prime}\right)+\lambda_{0}(x) \sum_{k=1}^{N} \xi_{i} \mu_{k k(N+1)(N+1), m n p q}^{\prime} & =-\sum_{j, k, l=1}^{N} \xi_{j} \partial_{k} u_{l}(x) \mu_{i j k l, m n p q}^{\prime},
\end{aligned}
$$


for all $p, q \in\{1, \ldots, N+1\}$ and $i, m, n \in\{1, \ldots, N\}$. In the same way, by (2.9) we obtain

$$
\xi_{k} \mu_{i j(N+1)(N+1), m n p q}^{\prime}=\xi_{i} \mu_{k j(N+1)(N+1), m n p q}^{\prime} \quad \text { on } \omega \times S^{N-1},
$$

for all $1 \leq i, j, k, m, n \leq N$ and $1 \leq p, q \leq N+1$.

Now, in order to obtain

$$
\sum_{p, q=1}^{N} \mu_{i j p q, p q(N+1)(N+1)}^{\prime}
$$

in terms of the $H$-measure $\mu$, we transform equality (2.21) twice by using (2.22). These computations are based on the fact that the $H$-measures have their supports included in $\mathbb{R}^{N} \times S^{N-1}$, and that equality (2.21) is satisfied for all $p, q \in\{1, \ldots, N+1\}$ and $i, m, n \in\{1, \ldots, N\}$.

First computation. By $(2.22)$ and since the $H$-measure $\mu^{\prime}$ has its support included in $\mathbb{R}^{N} \times S^{N-1}$, we have

$$
\left\{\begin{aligned}
\sum_{j, p=1}^{N} \xi_{j} \xi_{p} \mu_{j i(N+1)(N+1), m n p q}^{\prime} & =\sum_{j, p=1}^{N} \xi_{j}^{2} \mu_{p i(N+1)(N+1), m n p q}^{\prime}=\sum_{p=1}^{N} \mu_{p i(N+1)(N+1), m n p q}^{\prime} \\
\sum_{j, p=1}^{N} \xi_{p} \xi_{j} \mu_{i j(N+1)(N+1), m n p q}^{\prime} & =\sum_{j, p=1}^{N} \xi_{p} \xi_{i} \mu_{j j(N+1)(N+1), m n p q}^{\prime},
\end{aligned}\right.
$$

for all $q \in\{1, \ldots, N+1\}$ and $i, m, n \in\{1, \ldots, N\}$. Multiplying $(2.21)$ by $\xi_{p}$ and summing over $p \in\{1, \ldots, N\}$, the previous two equalities yield

$$
\begin{array}{r}
\mu_{0}\left(\sum_{j, p=1}^{N} \xi_{p} \xi_{i} \mu_{j j(N+1)(N+1), m n p q}^{\prime}+\sum_{p=1}^{N} \mu_{p i(N+1)(N+1), m n p q}^{\prime}\right)+\lambda_{0} \sum_{k, p=1}^{N} \xi_{i} \xi_{p} \mu_{k k(N+1)(N+1), m n p q}^{\prime} \\
=-\sum_{j, k, l, p=1}^{N} \xi_{j} \xi_{p} \partial_{k} u_{l}(x) \mu_{i j k l, m n p q}^{\prime}
\end{array}
$$

for all $q \in\{1, \ldots, N+1\}$ and $i, m, n \in\{1, \ldots, N\}$. Choosing $i=q$ and summing on $q$, equality (2.23) gives

$$
\begin{aligned}
\mu_{0}\left(\sum_{j, p, q=1}^{N} \xi_{p} \xi_{q} \mu_{j j(N+1)(N+1), m n p q}^{\prime}+\sum_{p, q=1}^{N} \mu_{p q(N+1)(N+1), m n p q}^{\prime}\right) & +\lambda_{0} \sum_{k, p, q=1}^{N} \xi_{q} \xi_{p} \mu_{k k(N+1)(N+1), m n p q}^{\prime} \\
& =-\sum_{j, k, l, p, q=1}^{N} \xi_{j} \xi_{p} \partial_{k} u_{l}(x) \mu_{q j k l, m n p q}^{\prime}
\end{aligned}
$$

for all $m, n \in\{1, \ldots, N\}$. Since $\mu_{i j k l, m n p q}^{\prime}=\mu_{i j k l, m n p q}$, for all $i, j, k, l, m, n, p, q \in\{1, \ldots, N\}$, we obtain

$$
\begin{aligned}
\sum_{p, q=1}^{N} \mu_{p q(N+1)(N+1), m n p q}^{\prime}= & -\frac{\lambda_{0}+\mu_{0}}{\mu_{0}} \sum_{j, p, q=1}^{N} \xi_{p} \xi_{q} \mu_{j j(N+1)(N+1), m n p q}^{\prime} \\
& -\frac{1}{\mu_{0}} \sum_{j, k, l, p, q=1}^{N} \xi_{j} \xi_{p} \partial_{k} u_{l}(x) \mu_{q j k l, m n p q},
\end{aligned}
$$

for all $m, n \in\{1, \ldots, N\}$. Now, in the previous equality, it remains to determine the first term of the right hand-side in terms of the $H$-measure $\mu$. 
Second computation. As in the previous computation, we have

$$
\left\{\begin{aligned}
\sum_{i, j=1}^{N} \xi_{j} \xi_{i} \mu_{j i(N+1)(N+1), m n p q}^{\prime} & =\sum_{i=1}^{N} \mu_{i i(N+1)(N+1), m n p q}^{\prime} \\
\sum_{i, j=1}^{N} \xi_{j} \xi_{i} \mu_{i j(N+1)(N+1), m n p q}^{\prime} & =\sum_{j=1}^{N} \mu_{j j(N+1)(N+1), m n p q}^{\prime}
\end{aligned}\right.
$$

for all $m, n \in\{1, \ldots, N\}$ and $p, q \in\{1, \ldots, N+1\}$. Multiplying equality (2.21) by $\xi_{i}$ and summing on $i$, this gives

$$
\begin{aligned}
\mu_{0}\left(\sum_{j=1}^{N} \mu_{j j(N+1)(N+1), m n p q}^{\prime}+\sum_{i=1}^{N} \mu_{i i(N+1)(N+1), m n p q}^{\prime}\right)+\lambda_{0} \sum_{i, k=1}^{N} \xi_{i}^{2} \mu_{k k(N+1)(N+1), m n p q}^{\prime} & =-\sum_{i, j, k, l=1}^{N} \xi_{i} \xi_{j} \partial_{k} u_{l}(x) \mu_{i j k l, m n p q}^{\prime},
\end{aligned}
$$

for all $m, n \in\{1, \ldots, N\}$ and $p, q \in\{1, \ldots, N+1\}$. Therefore

$$
\left(2 \mu_{0}+\lambda_{0}\right) \sum_{k=1}^{N} \mu_{k k(N+1)(N+1), m n p q}^{\prime}=-\sum_{i, j, k, l=1}^{N} \xi_{i} \xi_{j} \partial_{k} u_{l}(x) \mu_{i j k l, m n p q}^{\prime}
$$

for all $m, n \in\{1, \ldots, N\}$ and $p, q \in\{1, \ldots, N+1\}$. Multiplying the previous equality by $\xi_{p} \xi_{q}$ and summing on $p$ and $q$, we obtain

$$
\left(2 \mu_{0}+\lambda_{0}\right) \sum_{k, p, q=1}^{N} \xi_{p} \xi_{q} \mu_{k k(N+1)(N+1), m n p q}^{\prime}=-\sum_{i, j, k, l, p, q=1}^{N} \xi_{i} \xi_{j} \xi_{p} \xi_{q} \partial_{k} u_{l}(x) \mu_{i j k l, m n p q}^{\prime},
$$

for all $m, n \in\{1, \ldots, N\}$.

Third computation. From equalities (2.24) and (2.25) we deduce

$$
\begin{aligned}
\sum_{p, q=1}^{N} \mu_{p q(N+1)(N+1), m n p q}^{\prime}= & \frac{\mu_{0}+\lambda_{0}}{\mu_{0}\left(2 \mu_{0}+\lambda_{0}\right)} \sum_{i, j, k, l, p, q=1}^{N} \xi_{i} \xi_{j} \xi_{p} \xi_{q} \partial_{k} u_{l}(x) \mu_{i j k l, m n p q} \\
& -\frac{1}{\mu_{0}} \sum_{j, k, l, p, q=1}^{N} \xi_{j} \xi_{p} \partial_{k} u_{l}(x) \mu_{q j k l, m n p q},
\end{aligned}
$$

for all $m, n \in\{1, \ldots, N\}$. Since the $H$-measures are hermitian (see Rem. 2.2), equality (2.26) can be written

$$
\begin{aligned}
\sum_{p, q=1}^{N} \mu_{m n p q, p q(N+1)(N+1)}^{\prime}= & \frac{\mu_{0}+\lambda_{0}}{\mu_{0}\left(2 \mu_{0}+\lambda_{0}\right)} \sum_{i, j, k, l, p, q=1}^{N} \xi_{i} \xi_{j} \xi_{p} \xi_{q} \partial_{k} u_{l}(x) \mu_{m n p q, i j k l} \\
& -\frac{1}{\mu_{0}} \sum_{j, k, l, p, q=1}^{N} \xi_{j} \xi_{p} \partial_{k} u_{l}(x) \mu_{m n p q, q j k l},
\end{aligned}
$$


for all $m, n \in\{1, \ldots, N\}$. A suitable permutation of indices gives

$$
\begin{aligned}
\sum_{p, q=1}^{N} \mu_{i j p q, p q(N+1)(N+1)}^{\prime}= & \frac{\mu_{0}+\lambda_{0}}{\mu_{0}\left(2 \mu_{0}+\lambda_{0}\right)} \sum_{k, l, m, n, p, q=1}^{N} \xi_{m} \xi_{n} \xi_{p} \xi_{q} \partial_{k} u_{l} \mu_{i j p q, m n k l} \\
& -\frac{1}{\mu_{0}} \sum_{k, l, m, p, q=1}^{N} \xi_{m} \xi_{p} \partial_{k} u_{l} \mu_{i j p q, q m k l},
\end{aligned}
$$

for all $i, j \in\{1, \ldots, N\}$.

Third step. From (2.20) and (2.27) we deduce

$$
\begin{aligned}
\int_{\mathbb{R}^{N}}\left(\left(\mathbf{B}_{\varepsilon}-\mathbf{B}_{0}\right)(x) E_{\varepsilon}^{1}(x)\right)_{i j} \phi(x) \mathrm{d} x \underset{\varepsilon \rightarrow 0}{\longrightarrow} & \sum_{k, l, m, n, p, q=1}^{N}\left\langle\mu_{i j p q, m n k l}, \frac{\mu_{0}+\lambda_{0}}{\mu_{0}\left(2 \mu_{0}+\lambda_{0}\right)} \xi_{m} \xi_{n} \xi_{p} \xi_{q} \partial_{k} u_{l} \phi\right\rangle \\
& -\sum_{k, l, m, p, q=1}^{N}\left\langle\mu_{i j p q, q m k l}, \xi_{m} \xi_{p} \frac{\partial_{k} u_{l} \phi}{\mu_{0}}\right\rangle .
\end{aligned}
$$

Moreover, by (2.16) we have for all $\phi \in C_{c}(\omega)$,

$$
\begin{aligned}
\left\langle\left(\left(\mathbf{C}_{0}-\mathbf{C}^{*}\right) \nabla u\right)_{i j}, \phi\right\rangle= & \sum_{k, l, m, n, p, q=1}^{N}\left\langle\mu_{i j p q, m n k l}, \frac{\mu_{0}+\lambda_{0}}{\mu_{0}\left(2 \mu_{0}+\lambda_{0}\right)} \xi_{m} \xi_{n} \xi_{p} \xi_{q} \partial_{k} u_{l} \phi\right\rangle \\
& -\sum_{k, l, m, p, q=1}^{N}\left\langle\mu_{i j p q, q m k l}, \xi_{m} \xi_{p} \frac{\partial_{k} u_{l} \phi}{\mu_{0}}\right\rangle .
\end{aligned}
$$

Fix $k, l \in\{1, \ldots, N\}$. Let $\lambda$ be the matrix of coefficients defined by $\lambda_{i j}=0$ if $i \neq k$ or $j \neq l$, and $\lambda_{k l}=1$. We choose $u$ such that $\nabla u=\lambda$ on $\operatorname{supp}(\phi)$. Then, (2.28) reads

$$
\begin{aligned}
\left\langle\left(C^{*}-C_{0}\right)_{i j k l}, \phi\right\rangle= & -\sum_{m, n, p, q=1}^{N}\left\langle\mu_{i j p q, m n k l}, \xi_{m} \xi_{n} \xi_{p} \xi_{q} \frac{\mu_{0}+\lambda_{0}}{\mu_{0}\left(2 \mu_{0}+\lambda_{0}\right)} \phi\right\rangle \\
& +\sum_{m, p, q=1}^{N}\left\langle\mu_{i j p q, q m k l}, \frac{\phi}{\mu_{0}} \xi_{m} \xi_{p}\right\rangle .
\end{aligned}
$$

Finally, $\mathbf{C}^{*}=\mathbf{C}_{0}-\mathbf{M}$, where $\mathbf{M}$ has its coefficients given by

$$
\begin{aligned}
\left\langle M_{i j k l}, \phi\right\rangle= & -\sum_{m, n, p, q=1}^{N}\left\langle\mu_{i j p q, m n k l}, \xi_{m} \xi_{n} \xi_{p} \xi_{q} \frac{\mu_{0}+\lambda_{0}}{\mu_{0}\left(2 \mu_{0}+\lambda_{0}\right)} \phi\right\rangle \\
& +\sum_{m, p, q=1}^{N}\left\langle\mu_{i j p q, q m k l}, \xi_{m} \xi_{p} \frac{\phi}{\mu_{0}}\right\rangle,
\end{aligned}
$$

for all $i, j, k, l \in\{1, \ldots, N\}$ and all $\phi \in C_{c}(\Omega)$.

\subsubsection{Proof of formula (2.7)}

To prove formula (2.7), we first note that the coefficients of $\mathbf{B}_{\varepsilon}-\mathbf{B}_{0}$ are given by

$$
\left(B_{\varepsilon}-B_{0}\right)_{i j p q}=\left(\mu_{1}^{\varepsilon}-\mu_{1}\right)\left(\delta_{i p} \delta_{j q}+\delta_{i q} \delta_{j p}\right)+\left(\lambda_{1}^{\varepsilon}-\lambda_{1}\right) \delta_{i j} \delta_{p q},
$$


for all $i, j, p, q \in\{1, \ldots, N\}$. Then, if $\mu$ denotes the $H$-measure associated with the sequence $\left(\mathbf{B}_{\varepsilon}-\mathbf{B}_{0}\right)$ and $\nu$ the $H$-measure associated with the sequence $\left(\mu_{1}^{\varepsilon}-\mu_{1}, \lambda_{1}^{\varepsilon}-\lambda_{1}\right)$, we have

$$
\begin{aligned}
\mu_{i j p q, m n k l}= & \left(\delta_{i p} \delta_{j q} \delta_{m k} \delta_{n l}+\delta_{i q} \delta_{j p} \delta_{m k} \delta_{n l}+\delta_{i p} \delta_{j q} \delta_{m l} \delta_{n k}+\delta_{i q} \delta_{j p} \delta_{m l} \delta_{n k}\right) \nu_{11} \\
& +\left(\delta_{i j} \delta_{p q} \delta_{m n} \delta_{k l}\right) \nu_{22}+\left(\delta_{i p} \delta_{j q} \delta_{m n} \delta_{k l}+\delta_{i q} \delta_{j p} \delta_{m n} \delta_{k l}\right) \nu_{12} \\
& +\left(\delta_{m k} \delta_{n l} \delta_{i j} \delta_{p q}+\delta_{m l} \delta_{n k} \delta_{i j} \delta_{p q}\right) \nu_{21}
\end{aligned}
$$

for all $i, j, k, l, m, n, p, q \in\{1, \ldots, N\}$. Indeed, from (2.1) we have for any $\phi_{1}, \phi_{2} \in C_{c}(\Omega), \psi \in C\left(S^{N-1}\right)$

$$
\left\langle\mu_{i j p q, m n k l}, \phi_{1} \bar{\phi}_{2} \otimes \psi\right\rangle=\lim _{\varepsilon \rightarrow 0} \int_{\mathbb{R}^{N}} \mathcal{F}\left(\left(B_{\varepsilon}-B_{0}\right)_{i j p q} \phi_{1}\right) \overline{\mathcal{F}\left(\left(B_{\varepsilon}-B_{0}\right)_{m n k l} \phi_{2}\right)} \psi\left(\frac{\xi}{|\xi|}\right) \mathrm{d} \xi
$$

for all $i, j, k, l, m, n, p, q \in\{1, \ldots, N\}$, which by (2.30) gives, for example, for the last term

$$
\begin{aligned}
& \lim _{\varepsilon \rightarrow 0} \int_{\mathbb{R}^{N}} \mathcal{F}\left(\left(\lambda_{1}^{\varepsilon}-\lambda_{1}\right) \delta_{i j} \delta_{p q} \phi_{1}\right) \overline{\mathcal{F}\left(\left(\lambda_{1}^{\varepsilon}-\lambda_{1}\right) \delta_{m n} \delta_{k l} \phi_{2}\right)} \psi\left(\frac{\xi}{|\xi|}\right) \mathrm{d} \xi \\
& \quad=\delta_{i j} \delta_{p q} \delta_{m n} \delta_{k l} \lim _{\varepsilon \rightarrow 0} \int_{\mathbb{R}^{N}} \mathcal{F}\left(\left(\lambda_{1}^{\varepsilon}-\lambda_{1}\right) \phi_{1}\right) \overline{\mathcal{F}\left(\left(\lambda_{1}^{\varepsilon}-\lambda_{1}\right) \phi_{2}\right)} \psi\left(\frac{\xi}{|\xi|}\right) \mathrm{d} \xi \\
& \quad=\delta_{i j} \delta_{p q} \delta_{m n} \delta_{k l}\left\langle\nu_{22}, \phi_{1} \bar{\phi}_{2} \otimes \psi\right\rangle .
\end{aligned}
$$

Moreover, using the change of variable $\xi^{\prime}=-\xi$, we also have

$$
\begin{aligned}
\left\langle\xi_{i} \xi_{j} \nu_{12}, \phi_{1} \overline{\phi_{2}}\right\rangle & =\lim _{\varepsilon \rightarrow 0} \int_{\mathbb{R}^{N}} \mathcal{F}\left(\left(\mu_{1}^{\varepsilon}-\mu_{1}\right) \phi_{1}\right)(\xi) \overline{\mathcal{F}\left(\left(\lambda_{1}^{\varepsilon}-\lambda_{1}\right) \phi_{2}\right)(\xi)} \frac{\xi_{i} \xi_{j}}{|\xi|^{2}} \mathrm{~d} \xi \\
& =\lim _{\varepsilon \rightarrow 0} \int_{\mathbb{R}^{N}} \overline{\mathcal{F}\left(\left(\mu_{1}^{\varepsilon}-\mu_{1}\right) \phi_{1}\right)\left(\xi^{\prime}\right)} \mathcal{F}\left(\left(\lambda_{1}^{\varepsilon}-\lambda_{1}\right) \phi_{2}\right)\left(\xi^{\prime}\right) \frac{\xi_{i} \xi_{j}}{|\xi|^{2}} \mathrm{~d} \xi^{\prime} \\
& =\overline{\left\langle\xi_{i} \xi_{j} \nu_{12}, \phi_{1} \overline{\phi_{2}}\right\rangle},
\end{aligned}
$$

for all $i, j \in\{1, \ldots, N\}$. Since the $H$-measures are hermitian, we deduce that, for all $i, j \in\{1, \ldots, N\}$,

$$
\xi_{i} \xi_{j} \nu_{12}=\xi_{i} \xi_{j} \operatorname{Re}\left(\nu_{12}\right)=\xi_{i} \xi_{j} \nu_{21}
$$

Now from (2.31) we obtain, for all $i, j, k, l \in\{1, \ldots, N\}$,

$$
\begin{aligned}
\sum_{m, p, q=1}^{N} \xi_{m} \xi_{p} \mu_{i j p q, q m k l}= & \sum_{m, p, q=1}^{N}\left(\delta_{i p} \delta_{j q} \delta_{q k} \delta_{m l}+\delta_{i q} \delta_{j p} \delta_{q k} \delta_{m l}+\delta_{i p} \delta_{j q} \delta_{q l} \delta_{m k}+\delta_{i q} \delta_{j p} \delta_{q l} \delta_{m k}\right) \xi_{m} \xi_{p} \nu_{11} \\
& +\sum_{m, p, q=1}^{N}\left(\delta_{i p} \delta_{j q} \delta_{q m} \delta_{k l}+\delta_{i q} \delta_{j p} \delta_{q m} \delta_{k l}+\delta_{q k} \delta_{m l} \delta_{i j} \delta_{p q}+\delta_{q l} \delta_{m k} \delta_{i j} \delta_{p q}\right) \xi_{m} \xi_{p} \operatorname{Re}\left(\nu_{12}\right) \\
& +\sum_{m, p, q=1}^{N}\left(\delta_{i j} \delta_{p q} \delta_{q m} \delta_{k l}\right) \xi_{m} \xi_{p} \nu_{22} \\
= & \left(\xi_{i} \xi_{l} \delta_{j k}+\xi_{j} \xi_{l} \delta_{i k}+\xi_{i} \xi_{k} \delta_{j l}+\xi_{j} \xi_{k} \delta_{i l}\right) \nu_{11}+\delta_{i j} \delta_{k l}|\xi|^{2} \nu_{22}+2\left(\delta_{k l} \xi_{i} \xi_{j}+\delta_{i j} \xi_{k} \xi_{l}\right) \operatorname{Re}\left(\nu_{12}\right)
\end{aligned}
$$

and similarly

$$
\sum_{m, n, p, q=1}^{N} \xi_{m} \xi_{n} \xi_{p} \xi_{q} \mu_{i j p q, m n k l}=4 \xi_{i} \xi_{j} \xi_{k} \xi_{l} \nu_{11}+\delta_{i j} \delta_{k l}|\xi|^{4} \nu_{22}+2\left(\delta_{k l}|\xi|^{2} \xi_{i} \xi_{j}+\delta_{i j}|\xi|^{2} \xi_{k} \xi_{l}\right) \operatorname{Re}\left(\nu_{12}\right)
$$


Since the $H$-measures have their supports included in $\mathbb{R}^{N} \times S^{N-1}$, we obtain

$$
|\xi|^{2} \xi_{i} \xi_{j} \operatorname{Re}\left(\nu_{12}\right)=\xi_{i} \xi_{j} \operatorname{Re}\left(\nu_{12}\right) \quad \text { and } \quad\left\langle|\xi|^{2} \nu_{22}, \phi\right\rangle=\left\langle|\xi|^{4} \nu_{22}, \phi\right\rangle=\left\langle\nu_{22}, \phi \otimes 1\right\rangle, \forall \phi \in C_{c}(\Omega) \text {. }
$$

Then, using (2.32) and (2.33), formula (2.6) leads us to (2.7).

\section{Simplified MODELS IN CONDUCTION}

\subsection{Statement of the results}

In the sequel, $\theta:=\pi r^{2}$.

Theorem 3.1. We set

$$
A_{\varepsilon}^{I}:=\left(a\left(1-\chi_{\varepsilon}^{I}\right)+b \chi_{\varepsilon}^{I}\right) I_{3},
$$

where $b:=a+c \delta$ with $\delta>0$ small enough, $a>0$ and $c \in \mathbb{R}$. Then $A_{\varepsilon}^{I} H$-converges to $A_{\mathrm{eff}}^{I}$ which satisfies

$$
A_{\mathrm{eff}}^{I}=\left(a(1-\theta)+b \theta-\frac{(b-a)^{2}}{2 a} \theta(1-\theta)\right) I_{3}+\frac{(b-a)^{2}}{2 a} \theta(1-\theta)(\tau \otimes \tau)+o\left(\delta^{2}\right),
$$

where $\tau$ is given by (1.1).

Remark 3.2. Formula (3.2) shows the validity of the model $A_{\mathrm{eff}}^{I}=\alpha I_{3}+\beta(\tau \otimes \tau)$ (at the second order) under the small amplitude assumption. Thus this model validates rigorously the biomechanics one in the conduction case.

For the second model, the computations cannot be as much simplified as for the first model. To compare this model with model (0.3), we compute the orthogonal projection of the effective matrix $A_{\text {eff }}^{I I}$ on the space of the matrices of the form (0.3), i.e. the space $\left\{\alpha I_{3}+\beta(\tau \otimes \tau) \mid \alpha, \beta \in \mathbb{R}\right\}$.

Notation 3.3. Let $N \in \mathbb{N}$ and let $E$ be a linear subspace of $\mathbb{R}^{N \times N}$, we denote by $E^{\perp}$ the orthogonal subspace of E, i.e.

$$
E^{\perp}:=\left\{A \in \mathbb{R}^{N \times N} \mid A: B=0, \forall B \in E\right\} .
$$

For any matrix $A \in \mathbb{R}^{N \times N}$, there is a unique orthogonal decomposition

$$
A=A_{1} \oplus A_{2}, \quad \text { with } A_{1} \in E \text { and } A_{2} \in E^{\perp} \text {. }
$$

For the second small amplitude model we have the following result:

Theorem 3.4. We set

$$
A_{\varepsilon}^{I I}:=\left(a\left(1-\chi_{\varepsilon}^{I I}\right)+b \chi_{\varepsilon}^{I I}\right) I_{3},
$$

where $b:=a+c \delta$ with $\delta>0$ small enough, $a>0$ and $c \in \mathbb{R}$. Then $A_{\varepsilon}^{I I} H$-converges to $A_{\mathrm{eff}}^{I I}$ which admits the orthogonal decomposition

where $D_{\text {eff }}$ is a matrix-valued function satisfying

$$
A_{\mathrm{eff}}^{I I}(x)=A_{\mathrm{eff}}^{I}(x) \oplus \frac{(b-a)^{2}}{a} D_{\mathrm{eff}}(x)+o\left(\delta^{2}\right),
$$

$$
d(x):=\gamma^{\prime}\left(x_{1}\right)\left(\cos \gamma\left(x_{1}\right) x_{2}+\sin \gamma\left(x_{1}\right) x_{3}\right)=0 \Rightarrow D_{\text {eff }}(x)=0 .
$$

\section{Remark 3.5.}

1. The coefficients of the matrix-valued function $D_{\text {eff }}$ can be given but are not explicit.

2. If $\gamma^{\prime}\left(x_{1}\right)=0$ then $d(x)=0$. In some sense, the first model corresponds to the second one when the fiber orientation is locally constant.

3. Due to the extra term $D_{\text {eff }}(x)$, the second model does not coincide with the biomechanics one if $d(x) \neq 0$. 


\subsection{Proof of the results}

The proofs of Theorems 3.1 and 3.4 are based on formulas (1.4) and (1.8). Since the two proofs are similar we only give the one of Theorem 3.1 .

Proof of Theorem 3.1. We use the notations of Section 1.2.2. We have

$$
B_{\varepsilon}(x)=\left(a+c \chi_{\varepsilon}(x) \delta\right) I_{3} \quad \text { with } \quad \chi_{\varepsilon}(x):=\chi_{C}^{\#}\left(\frac{x}{\varepsilon}\right)
$$

Since $\chi_{\varepsilon}$ converges to $\theta$ weakly* in $L^{\infty}(\Omega)$, from Theorem 2.4 we deduce

$$
B_{\mathrm{eff}}=(a+(b-a) \theta) I_{3}-\frac{(b-a)^{2}}{a} M^{I}+o\left(\delta^{2}\right)
$$

where, by Notation 2.5,

$$
\left(M^{I}\right)_{i j}:=\sum_{k, l=1}^{3} \xi_{k} \xi_{l} \mu_{i k, l j} \quad i, j \in\{1,2,3\}
$$

and $\mu$ is the $H$-measure associated with the sequence $\left(\theta-\chi_{\varepsilon}\right) I_{3}$. If we denote by $\nu$ the $H$-measure associated with the sequence $\left(\theta-\chi_{\varepsilon}\right)$, we obtain by $(2.1)$ for all $\phi_{1}, \phi_{2} \in C_{c}(\Omega)$ and $\psi \in C\left(S^{2}\right)$

$$
\begin{aligned}
\left\langle\mu_{i k, l j}, \phi_{1} \bar{\phi}_{2} \otimes \psi\right\rangle & =\lim _{\varepsilon \rightarrow 0} \int_{\mathbb{R}^{3}} \mathcal{F}\left(\left(\theta-\chi_{\varepsilon}\right) \delta_{i k} \phi_{1}\right) \overline{\mathcal{F}\left(\left(\theta-\chi_{\varepsilon}\right) \delta_{l j} \phi_{2}\right)} \psi\left(\frac{\xi}{|\xi|}\right) \mathrm{d} \xi \\
& =\delta_{i k} \delta_{l j} \lim _{\varepsilon \rightarrow 0} \int_{\mathbb{R}^{3}} \mathcal{F}\left(\left(\theta-\chi_{\varepsilon}\right) \phi_{1}\right) \overline{\mathcal{F}\left(\left(\theta-\chi_{\varepsilon}\right) \phi_{2}\right)} \psi\left(\frac{\xi}{|\xi|}\right) \mathrm{d} \xi \\
& =\left\langle\delta_{i k} \delta_{l j} \nu, \phi_{1} \phi_{2} \otimes \psi\right\rangle,
\end{aligned}
$$

for all $i, j, k, l=1,2,3$. Then, for all $i, j, k, l=1,2,3$,

$$
\mu_{i k, l j}=\delta_{i k} \delta_{l j} \nu
$$

which implies

$$
\left(M^{I}\right)_{i j}=\xi_{i} \xi_{j} \nu
$$

Finally, using the periodicity of $\chi_{C}^{\#}$, we deduce from Proposition 2.3

$$
\left(M^{I}\right)_{i j}=\sum_{k \in \mathbb{Z}^{3} \backslash\{0\}}|\hat{\chi}(k)|^{2} \frac{k_{i} k_{j}}{|k|^{2}}, \quad \text { with } \quad \hat{\chi}(k):=\int_{Y_{3}} \chi_{C}^{\#}(x) \mathrm{e}^{-2 \mathrm{i} \pi x \cdot k} \mathrm{~d} x,
$$

for any $i, j=1,2,3$. The characteristic function $\chi_{C}^{\#}$ is independent of the $x_{2}$ variable, so

$$
\hat{\chi}\left(k_{1}, k_{2}, k_{3}\right)=0 \quad \text { if } \quad k_{2} \neq 0,
$$

whence

Furthermore,

$$
\left(M^{I}\right)_{22}=\left(M^{I}\right)_{12}=\left(M^{I}\right)_{21}=\left(M^{I}\right)_{32}=\left(M^{I}\right)_{23}=0
$$

which gives

$$
\hat{\chi}\left(k_{1}, 0, k_{3}\right)=\hat{\chi}\left(k_{3}, 0, k_{1}\right)=\hat{\chi}\left(-k_{1}, 0, k_{3}\right),
$$

We also have

$$
\left(M^{I}\right)_{13}=\left(M^{I}\right)_{31}=0
$$

$$
\left(M^{I}\right)_{11}=\left(M^{I}\right)_{33},
$$


since $\hat{\chi}\left(k_{1}, 0, k_{3}\right)=\hat{\chi}\left(k_{3}, 0, k_{1}\right)$. Then, we obtain

$$
\begin{aligned}
\left\langle\left(M^{I}\right)_{11},|\phi|^{2}\right\rangle & =\left\langle\frac{1}{2}\left(\left(M^{I}\right)_{11}+\left(M^{I}\right)_{22}+\left(M^{I}\right)_{33}\right),|\phi|^{2}\right\rangle \\
& =\left\langle\frac{1}{2}\left(\xi_{1}^{2}+\xi_{2}^{2}+\xi_{3}^{2}\right) \nu,|\phi|^{2}\right\rangle=\frac{1}{2}\left\langle\nu,|\phi|^{2} \otimes 1\right\rangle \\
\left\langle\left(M^{I}\right)_{11},|\phi|^{2}\right\rangle & =\frac{1}{2} \theta(1-\theta) \int_{\mathbb{R}^{3}}|\phi(x)|^{2} \mathrm{~d} x .
\end{aligned}
$$

Thus, $\left(M^{I}\right)_{11}=\left(M^{I}\right)_{33}=\frac{\theta(1-\theta)}{2}$ and

$$
B_{\mathrm{eff}}=((1-\theta) a+\theta b) I_{3}-\frac{(b-a)^{2}}{2 a} \theta(1-\theta)\left(I_{3}-e_{2} \otimes e_{2}\right)+o\left(\delta^{2}\right) .
$$

From (1.4) and (3.9), we have

$$
A_{\mathrm{eff}}^{I}(x)=(a(1-\theta)+b \theta) I_{3}-\frac{(b-a)^{2}}{2 a} \theta(1-\theta)\left(I_{3}-R\left(x_{1}\right)^{T}\left(e_{2} \otimes e_{2}\right) R\left(x_{1}\right)\right)+o\left(\delta^{2}\right) .
$$

Moreover $R\left(x_{1}\right)^{T}\left(e_{2} \otimes e_{2}\right) R\left(x_{1}\right)=R\left(x_{1}\right)^{T} e_{2} \otimes R\left(x_{1}\right)^{T} e_{2}=\tau\left(x_{1}\right) \otimes \tau\left(x_{1}\right)$, which gives the result.

\section{Simplified MODELS IN ELASTICITY}

\subsection{Statement of the results}

\subsubsection{Models $I$ and $I I$}

For the first small amplitude model we have the following result:

Theorem 4.1. We set

$$
\mathbf{A}_{\varepsilon}^{I}:=\left(1-\chi_{\varepsilon}^{I}\right) \mathbf{A}^{1}+\chi_{\varepsilon}^{I} \mathbf{A}^{2},
$$

where $\mathbf{A}^{1}, \mathbf{A}^{2}$ are two homogeneous (i.e. constant) and isotropic fourth order tensor. Let $\nu_{x}$ be the $H$-measure associated with $\left(\theta-\chi_{\varepsilon}^{I}\right)$ and $\lambda_{1}, \mu_{1}$ be the Lamé coefficients of $\mathbf{A}^{1}$. We assume the Lamé coefficients $\mu_{2}$ and $\lambda_{2}$ of $\mathbf{A}^{2}$ read as

$$
\mu_{2}:=\mu_{1}+\delta \mu \quad \text { and } \quad \lambda_{2}:=\lambda_{1}+\delta \lambda,
$$

where $\delta>0$ is small enough and $\mu, \lambda \in \mathbb{R}$. Then $\mathbf{A}_{\varepsilon}^{I} H$-converges to $\mathbf{A}_{\text {eff }}^{I}$ which is given, for all $e \in \mathbb{R}_{s}^{3 \times 3}$, by

$$
\begin{aligned}
& \mathbf{A}_{\text {eff }}^{I}(x) e=\mathbf{A}^{1} e+\theta\left(\mathbf{A}^{2} e-\mathbf{A}^{1} e\right) \\
& -\delta^{2} \theta(1-\theta)\left[\frac{2 \mu^{2}}{\mu_{1}} e+\frac{2 \mu \lambda+\lambda^{2}}{2 \mu_{1}+\lambda_{1}} \operatorname{tr}(e) I_{3}-\frac{\lambda \mu}{2 \mu_{1}+\lambda_{1}}\left(\operatorname{tr}(e)(\tau \otimes \tau)+(e \tau \cdot \tau) I_{3}\right)-\frac{\mu^{2}}{\mu_{1}}(e(\tau \otimes \tau)+\tau \otimes e \tau)\right] \\
& +\delta^{2} \mathbf{N}^{I}(x) e+o\left(\delta^{2}\right), \quad(4.3)
\end{aligned}
$$

where $\tau:=\tau\left(x_{1}\right)$ (given by (1.1)) and $\mathbf{N}^{I}(x)$ is the fourth order tensor whose coefficients are

$$
\left(N^{I}(x)\right)_{i j k l}:=\frac{4\left(\mu_{1}+\lambda_{1}\right)}{2 \mu_{1}+\lambda_{1}} \frac{\mu^{2}}{\mu_{1}} \xi_{i} \xi_{j} \xi_{k} \xi_{l} \nu_{x}
$$

for all $i, j, k, l=1,2,3$ (see Notation 2.5 for the meaning of the last term). 


\section{Remark 4.2.}

1. The $H$-measure $\nu_{x}$ is parametrized by $x$ since there is no periodicity assumption.

2. In order to compare the expression of $\mathbf{A}_{\text {eff }}^{I}$ with (0.2), one can assume furthermore that $\mu$ is small enough. This assumption allows us to remove the term $\mathbf{N}^{I}$ which depends on the $H$-measure $\nu_{x}$. Then the second order term of expansion (4.3) reads as, for any $\mu$ small enough,

$$
-\theta(1-\theta) \frac{2 \mu \lambda+\lambda^{2}}{2 \mu_{1}+\lambda_{1}} \operatorname{tr}(e) I_{3}+\theta(1-\theta) \frac{\lambda \mu}{2 \mu_{1}+\lambda_{1}}\left[\operatorname{tr}(e)(\tau \otimes \tau)+(e \tau \cdot \tau) I_{3}\right]+o\left(\mu^{2}\right) .
$$

Therefore, because of the extra term in $(e \tau \cdot \tau) I_{3}$, formula (4.3) does not coincide (under the small amplitude assumption) with the biomechanics model, contrary to the conduction case.

3. In fact, we can only conclude that the linear elasticity framework combined with the small amplitude assumption does not agree with the empirical model. An alternative approach would be to start from nonlinear (hyperelastic) behaviour laws (see Holzapfel [14], Ogden [16], Spencer [19]) and then to linearize around the identity. This could allow us to preserve the extra anisotropic terms like $(e \tau \cdot \tau) I_{3}$ and $e(\tau \otimes \tau)+\tau \otimes e \tau$, which are rejected in the previous analysis. However, this more sophisticated approach combining hyperelasticity and non-periodic homogenization widely goes out of the setting of our present study, and would thus need a completely new work.

For the second small amplitude model we have the following result:

Theorem 4.3. We set

$$
\mathbf{A}_{\varepsilon}^{I I}:=\left(1-\chi_{\varepsilon}^{I I}\right) \mathbf{A}^{1}+\chi_{\varepsilon}^{I I} \mathbf{A}^{2}
$$

where $\mathbf{A}^{1}, \mathbf{A}^{2}$ are two homogeneous and isotropic fourth order tensor. Let $\nu_{x}^{\prime}$ be the H-measure associated with the sequence $\left(\theta-\chi_{\varepsilon}^{I I}\right)$ and $\lambda_{1}, \mu_{1}$ be the Lamé coefficients of $\mathbf{A}^{1}$. We assume that the Lamé coefficients $\mu_{2}$ and $\lambda_{2}$ of $\mathbf{A}^{2}$ read as

$$
\mu_{2}:=\mu_{1}+\delta \mu \quad \text { and } \quad \lambda_{2}:=\lambda_{1}+\delta \lambda,
$$

where $\delta>0$ is small enough and $\mu, \lambda \in \mathbb{R}$. Then, $\mathbf{A}_{\varepsilon}^{I I} H$-converges to $\mathbf{A}_{\text {eff }}^{I I}$ which is given, for all $e \in \mathbb{R}_{s}^{3 \times 3}$, by

$$
\mathbf{A}_{\mathrm{eff}}^{I I}(x) e=\mathbf{A}_{\mathrm{eff}}^{I}(x) e+\delta^{2}\left(\mathbf{N}^{I I}(x) e-\mathbf{N}^{I}(x) e+\mathbf{P}(x) e\right)+o\left(\delta^{2}\right),
$$

where $\mathbf{N}^{I}(x)$ is given by (4.4) and $\mathbf{N}^{I I}(x), \mathbf{P}(x)$ are the fourth order tensor whose coefficients are

$$
\left(N^{I I}(x)\right)_{i j k l}:=\frac{4\left(\mu_{1}+\lambda_{1}\right)}{2 \mu_{1}+\lambda_{1}} \frac{\mu^{2}}{\mu_{1}} \xi_{i} \xi_{j} \xi_{k} \xi_{l} \nu_{x}^{\prime}
$$

and

$$
\begin{aligned}
(P(x))_{i j k l}:= & \frac{\mu^{2}}{\mu_{1}}\left(\delta_{i k}\left(D_{\mathrm{eff}}(x)\right)_{j l}+\delta_{i l}\left(D_{\mathrm{eff}}(x)\right)_{j k}+\delta_{j k}\left(D_{\mathrm{eff}}(x)\right)_{i l}+\delta_{j l}\left(D_{\mathrm{eff}}(x)\right)_{i k}\right) \\
& +\frac{2 \lambda \mu}{2 \mu_{1}+\lambda_{1}}\left(\delta_{k l}\left(D_{\mathrm{eff}}(x)\right)_{i j}+\delta_{i j}\left(D_{\mathrm{eff}}(x)\right)_{k l}\right),
\end{aligned}
$$

for all $i, j, k, l=1,2,3$, with $D_{\mathrm{eff}}(x)$ the matrix-valued function given by (3.4).

Remark 4.4. The second order term of the expansion (4.7) reads, for any $\mu$ small enough,

$$
\begin{aligned}
-\theta(1-\theta) \frac{2 \mu \lambda+\lambda^{2}}{2 \mu_{1}+\lambda_{1}} \operatorname{tr}(e) I_{3}+\theta(1-\theta) \frac{\lambda \mu}{2 \mu_{1}+\lambda_{1}}\left(\operatorname{tr}(e)(\tau \otimes \tau)+(e \tau \cdot \tau) I_{3}\right) & \\
& +\frac{2 \lambda \mu}{2 \mu_{1}+\lambda_{1}}\left(\operatorname{tr}(e) D_{\mathrm{eff}}(x)+\left(D_{\mathrm{eff}}(x): e\right) I_{3}\right)+o\left(\mu^{2}\right) .
\end{aligned}
$$


Thus, from Remark 4.2, the difference between the two models at the second order is given for any $\mu$ small enough by

$$
\frac{2 \lambda \mu}{2 \mu_{1}+\lambda_{1}}\left(\operatorname{tr}(e) D_{\mathrm{eff}}(x)+\left(D_{\mathrm{eff}}(x): e\right) I_{3}\right)+o\left(\mu^{2}\right)
$$

In particular, the two models coincide when the fiber orientation is locally constant.

\subsubsection{Model III}

Since models $I$ and $I I$ do not valid the biomechanics one in linearized elasticity, we introduced a new model. From [5,6], we know that the first model in conduction locally corresponds to the periodic distribution of fibers of constant orientation. This can be easily extended to the elasticity case. Then we are led to a periodic microstructure with fibers of constant orientation $\tau$. As in the previous models, $\tau$ depends on $z_{1}$ but we omit this dependence. Then for this third model we focus on the anisotropy rather than on the non-periodicity.

We fix $z \in \mathbb{R}^{3}$. Set $\tau:=\tau\left(z_{1}\right)$ and $R:=R\left(z_{1}\right)$ defined by $(1.5)$. Let $\chi_{C}^{\#}$ be the $Y_{3}$-periodic function defined in $Y_{3}$ as the characteristic function of the cylinder $C$ given by (1.2). We have the following result:

Theorem 4.5. We set

$$
\mathbf{A}_{\varepsilon}^{I I I}:=\left(1-\chi_{\varepsilon}^{\tau}\right) \mathbf{A}^{1}+\chi_{\varepsilon}^{\tau} \mathbf{A}^{2} \quad \text { with } \quad \chi_{\varepsilon}^{\tau}(x):=\chi_{C}^{\#}\left(\frac{R x}{\varepsilon}\right),
$$

where $\mathbf{A}^{1}, \mathbf{A}^{2} \in \mathcal{M}^{4}(\alpha, \beta ; \Omega)$. We assume $\mathbf{A}^{1}$ is homogeneous and isotropic of Lamé coefficients $\mu_{1}, \lambda_{1}$ and $\mathbf{A}^{2}$ is given, for all $e \in \mathbb{R}_{s}^{3 \times 3}$, by

$$
\mathbf{A}^{2} e=\mathbf{A}^{1} e+\delta(e \tau \cdot \tau)(\tau \otimes \tau),
$$

where $\delta>0$ is small enough. Then, $\mathbf{A}_{\varepsilon}^{I I I} H$-converges to $\mathbf{A}_{\mathrm{eff}}^{I I I}$ which is given, for all $e \in \mathbb{R}_{s}^{3 \times 3}$, by

$$
\mathbf{A}_{\mathrm{eff}}^{I I I} e=\mathbf{A}^{1} e+\theta\left(\mathbf{A}^{2} e-\mathbf{A}^{1} e\right)-\kappa_{\nu, \tau} \delta^{2} \frac{\mu_{1}+\lambda_{1}}{\mu_{1}\left(2 \mu_{1}+\lambda_{1}\right)}(e \tau \cdot \tau)(\tau \otimes \tau)+o\left(\delta^{2}\right),
$$

with $\kappa_{\nu, \tau}$ given by (see Notation 2.5)

$$
\kappa_{\nu, \tau}=\sum_{m, n, p, q=1}^{3} \tau_{m} \tau_{n} \tau_{p} \tau_{q} \xi_{m} \xi_{n} \xi_{p} \xi_{q} \nu^{\tau}
$$

where $\nu^{\tau}$ is the $H$-measure associated with the sequence $\left(\theta-\chi_{\varepsilon}^{\tau}\right)$.

\section{Remark 4.6.}

1. Formula (4.12) shows the validity of the model $\lambda \operatorname{tr}(e)+2 \mu e+\kappa(e \tau \cdot \tau)(\tau \otimes \tau)$ (at the second order) under the small amplitude assumption.

2. From (4.13) and Proposition 2.3, we have that $\kappa_{\nu, \tau}$ is constant. Moreover, by Notation 2.5, equality (4.13) reads as, for all $\phi \in C_{c}(\Omega)$ such that $\int_{\Omega} \phi \mathrm{d} x=1$,

$$
\kappa_{\nu, \tau}=\sum_{m, n, p, q=1}^{3}\left(\tau_{m} \tau_{n} \tau_{p} \tau_{q}\right)\left\langle\nu^{\tau}, \phi \otimes \xi_{m} \xi_{n} \xi_{p} \xi_{q}\right\rangle
$$

Using Proposition 2.3 the constant $\kappa_{\nu, \tau}$ may be written as a series. For example, in the case $\tau=e_{2}$, a simple computation leads to

$$
\kappa_{\nu, e_{2}}=\sum_{k \in \mathbb{Z}^{3} \backslash\{0\}}\left|\hat{\chi}_{2}(k)\right|^{2} \frac{k_{2}^{4}}{|k|^{4}} \quad \text { with } \quad \hat{\chi}_{2}(k)=\frac{1}{\left|Y_{3}\right|} \int_{Y_{3}} \chi_{\varepsilon}^{e_{2}}(y) \mathrm{e}^{-2 \mathrm{i} \pi k \cdot y} \mathrm{~d} y
$$

which cannot be easily simplified. 
In the same way, one can obtain an effective matrix with a zero $H$-correction.

Proposition 4.7. Consider $\mathbf{A}_{\varepsilon}^{I I I}$ defined by (4.10) with $\mathbf{A}^{1}$ homogeneous and isotropic and $\mathbf{A}^{2}$ given, for all $e \in \mathbb{R}_{s}^{3 \times 3}$, by

$$
\mathbf{A}^{2} e=\mathbf{A}^{1} e+\delta \operatorname{tr}(e)(\tau \otimes \tau),
$$

where $\delta>0$ is small enough. Then, $\mathbf{A}_{\varepsilon}^{I I I} H$-converges to $\mathbf{A}_{\text {eff }}^{I I I}$ which is given, for all $e \in \mathbb{R}_{s}^{3 \times 3}$, by

$$
\mathbf{A}_{\mathrm{eff}}^{I I I} e=\mathbf{A}^{1} e+\theta\left(\mathbf{A}^{2} e-\mathbf{A}^{1} e\right)+o\left(\delta^{2}\right) .
$$

\subsection{Proof of the results}

Since the proofs of Theorems 4.1 and 4.3 are based on the same arguments, we only give the proof of Theorem 4.1. The proof of Theorem 4.5 is similar but with some specifics which are clarified.

Proof of Theorem 4.1. The formula (1.4) obtained for the first model is no more valid. Indeed, in elasticity the isotropy does not allow us to simplify the change of variable as in (1.4). So to obtain an expansion of $\mathbf{A}_{\text {eff }}^{I}$, we will use (3.2) and Theorem 2.7.

In the conduction case, $A_{\varepsilon}^{I}$ is given by

$$
A_{\varepsilon}^{I}=a I_{3}+c \delta \chi_{\varepsilon}^{I} I_{3}
$$

From the definition of $\chi_{\varepsilon}^{I}$ and by application of Lemma 2.6 of [5] we obtain that $\chi_{\varepsilon}^{I}$ converges weakly* in $L^{\infty}(\Omega)$ to $\theta=\pi r^{2}$. Thus the sequence $\left(\theta-\chi_{\varepsilon}^{I}\right)$ defines an $H$-measure $\nu_{x}$. Then, from Theorem 2.4 we deduce

$$
A_{\mathrm{eff}}^{I}(x)=a I_{3}+c \delta \theta I_{3}-M^{I}(x) \delta^{2}+o\left(\delta^{2}\right),
$$

where, for all $i, j=1,2,3$,

$$
\left(M^{I}\right)_{i j}(x):=\frac{c^{2}}{a} \xi_{i} \xi_{j} \nu_{x}
$$

On the other hand, by Theorem 3.1 we have

$$
A_{\mathrm{eff}}^{I}=a I_{3}+c \delta \theta I_{3}-\delta^{2} \frac{c^{2}}{a} \frac{\theta(1-\theta)}{2}\left(I_{3}-\tau \otimes \tau\right)+o\left(\delta^{2}\right) .
$$

Therefore, for all $i, j=1,2,3$,

$$
\xi_{i} \xi_{j} \nu_{x}=\frac{\theta(1-\theta)}{2}\left(\delta_{i j}-\tau_{i}(x) \tau_{j}(x)\right) .
$$

Recall that by Notation 2.5, (4.16) reads, for all $i, j=1,2,3$,

$$
\forall \phi \in C_{c}(\Omega), \quad\left\langle\nu_{x}, \phi \otimes \xi_{i} \xi_{j}\right\rangle=\frac{\theta(1-\theta)}{2} \int_{\Omega}\left(\delta_{i j}-\tau_{i}(x) \tau_{j}(x)\right) \phi(x) \mathrm{d} x .
$$

In linearized elasticity, we have by (4.1) and (4.2)

$$
\mathbf{A}_{\varepsilon}^{I}(x)=\lambda_{1}\left(I_{3} \otimes I_{3}\right)+2 \mu_{1} \mathbf{I}+\delta \chi_{\varepsilon}^{I}(x)\left(\lambda\left(I_{3} \otimes I_{3}\right)+2 \mu \mathbf{I}\right) .
$$

Then, by Theorem 2.7, $\mathbf{A}_{\varepsilon}^{I} H$-converges to $\mathbf{A}_{\text {eff }}^{I}$ which reads as

$$
\mathbf{A}_{\text {eff }}^{I}(x)=\lambda_{1}\left(I_{3} \otimes I_{3}\right)+2 \mu_{1} \mathbf{I}+\delta \theta\left(\lambda\left(I_{3} \otimes I_{3}\right)+2 \mu \mathbf{I}\right)-\delta^{2} \mathbf{M}^{I}(x)+o\left(\delta^{2}\right),
$$


where by (2.7) the coefficients of $\mathbf{M}^{I}(x)$ are given by

$$
\begin{aligned}
\left(M^{I}(x)\right)_{i j k l}= & \frac{\mu^{2}}{\mu_{1}}\left(\delta_{i k} \xi_{j} \xi_{l} \nu_{x}+\delta_{i l} \xi_{j} \xi_{k} \nu_{x}+\delta_{j k} \xi_{i} \xi_{l} \nu_{x}+\delta_{j l} \xi_{i} \xi_{k} \nu_{x}\right) \\
& +\frac{2 \lambda \mu}{2 \mu_{1}+\lambda_{1}}\left(\delta_{k l} \xi_{i} \xi_{j} \nu_{x}+\delta_{k l} \xi_{k} \xi_{l} \nu_{x}\right)+\theta(1-\theta) \frac{\lambda^{2}}{2 \mu_{1}+\lambda_{1}} \delta_{i j} \delta_{k l} \\
& -\frac{4\left(\mu_{1}+\lambda_{1}\right)}{2 \mu_{1}+\lambda_{1}} \frac{\mu^{2}}{\mu_{1}} \xi_{i} \xi_{j} \xi_{k} \xi_{l} \nu_{x},
\end{aligned}
$$

for all $i, j, k, l=1,2,3$. We use (4.16) to determine explicitly the first terms of $\mathbf{M}^{I}(x)$. From (4.16) and (4.18) we deduce that, for all $i, j=1,2,3$,

$$
\begin{aligned}
\left(\mathbf{M}^{I}(x) e\right)_{i j}= & \theta(1-\theta) \frac{2 \mu^{2}}{\mu_{1}} e_{i j}+\theta(1-\theta) \frac{2 \mu \lambda+\lambda^{2}}{2 \mu_{1}+\lambda_{1}} \operatorname{tr}(e) \delta_{i j} \\
& -\frac{\theta(1-\theta)}{2} \frac{\mu^{2}}{\mu_{1}}\left(2(e \tau)_{i} \tau_{j}+2(e \tau)_{j} \tau_{i}\right) \\
& -\theta(1-\theta) \frac{\lambda \mu}{2 \mu_{1}+\lambda_{1}}\left(\operatorname{tr}(e) \tau_{i} \tau_{j}+(e \tau \cdot \tau) \delta_{i j}\right)-\left(\mathbf{N}^{I}(x) e\right)_{i j}
\end{aligned}
$$

where $\mathbf{N}^{I}(x)$ is given by (4.4). This implies

$$
\begin{aligned}
\mathbf{M}^{I}(x) e= & \theta(1-\theta) \frac{2 \mu^{2}}{\mu_{1}} e+\theta(1-\theta) \frac{2 \mu \lambda+\lambda^{2}}{2 \mu_{1}+\lambda_{1}} \operatorname{tr}(e) I_{3} \\
& -\theta(1-\theta) \frac{\mu^{2}}{\mu_{1}}(e(\tau \otimes \tau)+\tau \otimes e \tau) \\
& -\theta(1-\theta) \frac{\lambda \mu}{2 \mu_{1}+\lambda_{1}}\left(\operatorname{tr}(e)(\tau \otimes \tau)+(e \tau \cdot \tau) I_{3}\right)-\mathbf{N}^{I}(x) e .
\end{aligned}
$$

Then (4.17) and (4.19) yield the desired result.

Remark 4.8. The last term is more complicated. For example, consider the simpler case of a periodic microstructure. Let $\chi_{C}^{\#}$ be the $Y_{3}$-periodic function defined on $Y_{3}$ as the characteristic function of the cylinder $C$ defined by $(1.2)$ and set $\chi_{\varepsilon}(x):=\chi_{C}^{\#}\left(\frac{x}{\varepsilon}\right)$. Let $\nu$ be the $H$-measure associated with $\left(\theta-\chi_{\varepsilon}\right)$, then we obtain by Proposition 2.3 , for all $i, j, k, l=1,2,3$,

$$
\xi_{i} \xi_{j} \xi_{k} \xi_{l} \nu=\sum_{n \in \mathbb{Z}^{3} \backslash\{0\}}|\hat{\chi}(n)|^{2} \frac{n_{i} n_{j} n_{k} n_{l}}{|n|^{4}}, \quad \text { where } \hat{\chi}(n):=\int_{Y_{3}} \chi_{C}^{\#}(x) \mathrm{e}^{-2 \mathrm{i} \pi x \cdot n} \mathrm{~d} x .
$$

Thus the simplifications made in the conduction case on the terms $\xi_{i} \xi_{j} \nu$ cannot be performed here.

Proof of Theorem 4.5. First note that

$$
\sum_{k, l=1}^{3} \tau_{k} \tau_{l} \xi_{k} \xi_{l} \nu^{\tau}=0
$$

which is a straightforward consequence of (4.16). As in the proof of Theorem 4.3, by (4.10) and (4.11) combined with Theorem 2.7, we obtain that $\mathbf{A}_{\varepsilon}^{I I I} H$-converges to the constant tensor-valued function $\mathbf{A}_{\text {eff }}^{I I I}$ which satisfies

$$
\mathbf{A}_{\mathrm{eff}}^{I I I}=\mathbf{A}^{1}+\theta\left(\mathbf{A}^{2}-\mathbf{A}^{1}\right)-\delta^{2} \mathbf{M}^{I I I}+o\left(\delta^{2}\right)
$$


where by (2.6) and Notation 2.5 the coefficients of $\mathbf{M}^{I I I}$ are given by

$$
\begin{aligned}
M_{i j k l}^{I I I}= & \frac{1}{\mu_{1}} \sum_{m, p, q=1}^{3} \xi_{m} \xi_{p} \mu_{i j p q, q m k l}^{\tau} \\
& +\frac{\mu_{1}+\lambda_{1}}{\mu_{1}\left(2 \mu_{1}+\lambda_{1}\right)} \sum_{m, n, p, q=1}^{3} \xi_{m} \xi_{n} \xi_{p} \xi_{q} \mu_{i j p q, m n k l}^{\tau}
\end{aligned}
$$

for all $i, j, k, l=1,2,3$, with $\mu^{\tau}$ the $H$-measure associated with the sequence $\left(\theta-\chi_{\varepsilon}^{\tau}\right)(\tau \otimes \tau \otimes \tau \otimes \tau)$. From (4.11) and (2.1) we obtain, for all $i, j, k, l, m, n, p, q=1,2,3$,

$$
\mu_{i j p q, m n k l}^{\tau}=\tau_{i} \tau_{j} \tau_{p} \tau_{q} \tau_{m} \tau_{n} \tau_{k} \tau_{l} \nu^{\tau}
$$

which gives

$$
\begin{aligned}
M_{i j k l}^{I I I}= & \frac{\tau_{i} \tau_{j} \tau_{k} \tau_{l}}{\mu_{1}} \sum_{m, p, q=1}^{3} \tau_{q}^{2} \tau_{p} \tau_{m} \xi_{m} \xi_{p} \nu^{\tau} \\
& +\frac{\left(\mu_{1}+\lambda_{1}\right) \tau_{i} \tau_{j} \tau_{k} \tau_{l}}{\mu_{1}\left(2 \mu_{1}+\lambda_{1}\right)} \sum_{m, n, p, q=1}^{3} \tau_{m} \tau_{n} \tau_{p} \tau_{q} \xi_{m} \xi_{n} \xi_{p} \xi_{q} \nu^{\tau}
\end{aligned}
$$

Since $|\tau|=1$, we deduce from (4.20) that

$$
\sum_{m, p, q=1}^{3} \tau_{q}^{2} \tau_{p} \tau_{m} \xi_{m} \xi_{p} \nu^{\tau}=\sum_{m, p=1}^{3} \tau_{p} \tau_{m} \xi_{m} \xi_{p} \nu^{\tau}=0
$$

which gives

$$
M_{i j k l}^{I I I}=\tau_{i} \tau_{j} \tau_{k} \tau_{l} \frac{\left(\mu_{1}+\lambda_{1}\right)}{\mu_{1}\left(2 \mu_{1}+\lambda_{1}\right)} \kappa_{\nu, \tau},
$$

for all $i, j, k, l=1,2,3$, where $\kappa_{\nu, \tau}$ is given by (4.13).

Acknowledgements. The author wishes to thank M. Briane and the referees for many comments and suggestions. He is also grateful for support from ACI-NIM plan lepoumonvousdisje grant 2003-45.

\section{REFERENCES}

[1] G. Allaire, Shape Optimization by the Homogenization Method. Springer-Verlag, New York (2002).

[2] G. Allaire and S. Gutiérrez, Optimal design in small amplitude homogenization. R.I. 576, École Polytechnique, C.M.A UMRCNRS 7641 (2002).

[3] M.J. Arts, A Mathematical Model of the Dynamics of the Left Ventricle. Ph.D. thesis, University of Limburg, The Netherlands (1978).

[4] A. Bensoussan, J.-L. Lions and G. Papanicolaou, Asymptotic Analysis for periodic Structures. North-Holland (1978).

[5] M. Briane, Homogénéisation de materiaux fibrés et multi-couches. Ph.D. thesis, Université Paris 6, France (1990).

[6] M. Briane, Three models of non periodic fibrous materials obtained by homogenization. RAIRO Modél. Math. Anal. Numér. 27 (1993) 759-775.

[7] M. Briane, Homogenization of a nonperiodic material. J. Math. Pures Appl. 73 (1994) 47-66.

[8] D. Caillerie, A. Mourad and A. Raoult, Towards a fibre-based constitutive law for the myocardium. ESAIM: Proc. 12 (2002) $25-30$.

[9] D. Caillerie, A. Mourad and A. Raoult, Cell-to-muscle homogenization. Application to a constitutive law for the myocardium. ESAIM: M2AN 37 (2003) 681-698. 
[10] R.S. Chadwick, Mechanics of the left ventricle. Biophys J. 39 (1982) 279-288.

[11] T.S. Feit, Diastolic pressure-volume relations and distribution of pressure and fiber extension across the wall of a model of left ventricle. Biophys. J. 28 (1979) 143-166.

[12] G. Francfort and F. Murat, Homogenization and optimal bounds in linear elasticity. Arch. Rational Mech. Anal. 94 (1986) 307-334.

[13] P. Gérard, Microlocal defect measures. Comm. Partial Diff. Equations 16 (1991) 1761-1794.

[14] G.A. Holzapfel, Nonlinear solid mechanics. A continuum approach for engineering. John Wiley and Sons, Ltd., Chichester (2000).

[15] F. Murat and L. Tartar, H-convergence, in Topics in the Mathematical Modelling of Composite Materials, A.V. Cherkaev and R.V. Kohn Eds., Progress in Nonlinear Differential Equations and their Applications, Birkaüser, Boston (1998) $21-43$.

[16] R.W. Ogden, Nonlinear elasticity, anisotropy, material stability and residual stresses in soft tissue, in Biomechanics of Soft Tissue in Cardiovascular Systems, G.A. Holzapfel and R.W. Ogden Eds., CISM Courses and Lectures Series 441, Springer, Wien (2003) 65-108.

[17] C.S. Peskin, Fiber architecture of the left ventricular wall: an asymptotic analysis. Commun. Pure Appl. Math. 42 (1989) $79-113$.

[18] S. Spagnolo, Sulla convergenza di soluzioni di equazioni paraboliche ed ellitiche. Ann. Sc. Norm. Sup. Pisa 22 (1968) $571-597$.

[19] A.J.M. Spencer, Constitutive theory for strongly anisotropic solids, in Continuum Theory of the Mechanics of Fiber-Reinforced Composites, A.J.M. Spencer Ed., CISM Courses and Lectures Notes 282, International Center for Mechanical Sciences, Springer, Wien (1984) 1-32.

[20] D.D. Streeter, Gross morphology and fiber geometry of the heart, in Handbook of physiology. The cardiovascular system, R.M. Berne and N. Sperelakis Eds., Vol. 1, Williams and Wilkins, Baltimore (1979) 61-112.

[21] L. Tartar, H-measures and Small Amplitude Homogenization, in Random Media and Composites, R.V. Kohn and G.W. Milton Eds., SIAM, Philadelphia (1989) 89-99.

[22] L. Tartar, $H$-measures, a New approach for studying homogenization, oscillations and concentration effects in partial differential equations. Proc. R. Soc. Edin. 115-A (1990) 193-230.

[23] L. Tartar, An Introduction to the Homogenization Method in Optimal Design. Springer Lecture Notes Math. 1740 (2000) $47-156$. 